Chronic Obstructive Pulmonary Diseases: Journal of the COPD Foundation

\author{
Clinical Review
}

\title{
Systematic Review of the Association Between Laboratory- and Field-Based Exercise Tests and Lung Function in Patients with Chronic Obstructive Pulmonary Disease
}

\author{
Martin Bell, $\mathrm{PhD}^{1}$ Iain Fotheringham, $\mathrm{BA}^{1}$ Yogesh Suresh Punekar, $\mathrm{PhD}^{2}$ John H. Riley, BSc ${ }^{3}$ \\ Sarah Cockle, $\mathrm{PhD}^{2}$ Sally J. Singh, $\mathrm{PhD}^{3}$
}

\begin{abstract}
Introduction: Typical symptoms of chronic obstructive pulmonary disease (COPD) include breathlessness and reduced exercise capacity. Several laboratory- and field-based exercise tests are used to assess the exercise capacity of patients with COPD. It is unclear whether these exercise tests reflect the spirometric measures recommended for diagnosis of COPD. We therefore aimed to systematically assess the correlation between these exercise tests and common measures of lung function.

Methods: A search of Embase ${ }^{\mathrm{TM}}$, MEDLINE ${ }^{\circledR}$ and The Cochrane Library identified primary publications in English that reported data on the correlations (Pearson's $r$ or Spearman's rho) between the outcomes of exercise tests and the physiological measures of interest: forced expiratory volume in 1 second ( $\left.F E V_{1}\right)$, forced vital capacity, inspiratory capacity and arterial oxygen saturation. We included studies reporting on the following exercise tests: 6- and 12-minute walk tests (6MWT and 12MWT), incremental and endurance shuttle walk tests, incremental and endurance cycle ergometer tests, and treadmill tests.

Results: Of 1781 articles screened, 45 were ultimately deemed eligible for inclusion in this review. The most commonly reported lung function variable was $\mathrm{FEV}_{1}$ (reported by 39 studies); the most commonly reported exercise test was the 6-minute walk test (reported by 24 studies). FEV 1 appears to correlate moderately-to-strongly with $6 \mathrm{MWT}$ and 12MWT; and moderately-to-very strongly with incremental cycle ergometer tests (ICET); evidence for other exercise tests was limited.

Conclusion: There is evidence that 6MWT, 12MWT and ICET correlate with FEV 1 to some degree; evidence for associations of other exercise tests with measures of lung function in patients with COPD is limited. Clinicians must consider this when deciding to use these tests. Further comparisons of these tests must be made in order to assess which physiological and hemodynamic characteristics they reflect in patients with COPD.
\end{abstract}

\footnotetext{
Abbreviations: chronic obstructive pulmonary disease, COPD; forced expiratory volume in 1 second, FEV $\mathbf{1}$; 6-minute walk test, 6MWT; 12-minute walk test, 12MWT; incremental cycle ergometer test, ICET; Global initiative for chronic Obstructive Lung Disease, GOLD; American Thoracic Society, ATS; incremental shuttle walk test, ISWT; endurance shuttle walk test, ESWT; endurance cycle ergometer test, ECET; treadmill test, TT; forced vital capacity, FVC; inspiratory capacity, IC; partial pressure of arterial oxygen, $\mathbf{P a O}_{\mathbf{2}}$; Preferred Reporting Items for Systematic Reviews and Meta-Analyses, PRISMA; body mass index, BMI; arterial oxygen saturation (pulse oximetry), SaO $\mathbf{2}_{\mathbf{2}}$; liters, L; functional residual capacity, FRC; total lung capacity, TLC; residual volume, RV; arterial oxygen saturation, $\mathbf{S p O}_{\mathbf{2}}$; oxygen consumption, $\mathbf{V O}_{\mathbf{2}}$; heart rate, HR; health-related quality of life, HRQoL; BMI, airflow Obstruction, Dyspnea \& Exercise Capacity index, BODE; highest volume of oxygen consumption achieved, peak $\mathbf{V O}_{\mathbf{2}}$; highest workload achieved, $\mathbf{W}_{\text {max }}$; National Institute for Health Research, NIHR; Collaboration for Leadership in Applied Health Research and Care East Midlands, CLAHRC EM; National Health Service, NHS; British Thoracic Society, BTS; European Respiratory Society, ERS; interquartile range, IQR; inspiratory slow vital capacity, IVC; kilopascal, kPa; Medical Research Council, MRC; maximal voluntary ventilation, $\mathbf{M V V}$; not reported, $\mathbf{N R}$; partial pressure of arterial carbon dioxide, $\mathbf{P a C O}_{\mathbf{2}}$; respiratory exchange ratio, RE; standard deviation, SD; vital capacity, VC; correlation, Corr; studies reporting no significant correlation, NS; Spearman's rank coefficient, $\boldsymbol{\rho}$; Pearson's regression coefficient, $\boldsymbol{r}$
} 
Funding Support: The study was funded by GlaxoSmithKline, Uxbridge, United Kingdom

Date of Acceptance: March 20, 2015

Citation: Bell M, Fotheringham I, Punekar YS, Riley JH, Cockle S. Singh SJ. Systematic review of the association between laboratory- and field-based exercise tests and lung function in patients with chronic obstructive pulmonary disease. Chronic Obstr Pulm Dis (Miami). 2015; 2(4): 321-342. doi: http://dx.doi.org/10.12356/jcopdf. 2.4.2014.0157.

\section{This article has an online supplement}

1 Value Demonstration Practice, Oxford PharmaGenesis Ltd, Oxford, United Kingdom

2 Value Evidence \& Outcomes, GlaxoSmithKline, Brentford, United Kingdon

3 Global Clinical Development and Respiratory R\&D, GlaxoSmithKline, Uxbridge, United Kingdom

4 Centre for Exercise and Rehabilitation Science, University Hospitals of Leicester NHS Trust, Leicester, United Kingdom

\section{Address correspondence to:}

Yogesh Suresh Punekar, PhD

GlaxoSmithKline, Brentford, United Kingdom

yogesh.q.punekar@gsk.com

\section{Keywords:}

chronic obstructive pulmonary disease; COPD; systematic review; forced expiratory volume in 1 second; $F_{E V}$; exercise capacity

\section{Introduction}

Chronic obstructive pulmonary disease (COPD) is a leading cause of death worldwide and its global prevalence is projected to increase. ${ }^{1-3} \mathrm{COPD}$ is characterized by breathlessness, episodes of exacerbations and reduced exercise capacity. ${ }^{4}$ COPD can lead to a progressive loss of daily activities and increased sedentary behavior, further exacerbating exercise capacity impairment. ${ }^{5,6}$ Even in patients with mild COPD, physical activity ${ }^{7}$ and exercise performance ${ }^{8}$ are compromised, and exercise tolerance is increasingly attenuated with disease progression. ${ }^{7}$ The mechanisms underlying reduced exercise capacity in patients with COPD are varied, but include increased metabolic costs of breathing ${ }^{9}$; deficits in gas exchange and ventilatory mechanics ${ }^{6}$; and peripheral muscle dysfunction. ${ }^{8,10}$

Spirometry is recommended by the Global initiative for chronic Obstructive Lung Disease (GOLD) for the diagnosis of COPD. ${ }^{4}$ However, spirometry alone is a poor predictor of disability and quality of life in patients with $\mathrm{COPD}^{11,12}$ and correlates only weakly with dyspnea and health status. ${ }^{12,13,14}$ In contrast, exercise test outcomes have been shown to have good prognostic capabilities in patients with COPD. ${ }^{15-21}$ Guidelines published by the National Institute for Clinical Excellence and the American Thoracic Society (ATS)/European Respiratory Society on the diagnosis and treatment of COPD now indicate that prognosis and assessment of disease severity is improved by using functional criteria such as exercise capacity. ${ }^{22,23}$ Furthermore, the European Medicines Agency also supports the assertion that exercise testing in the clinical setting is a useful tool in COPD prognosis and monitoring the effectiveness of therapeutic intervention. $^{24}$

Several test modalities are available for the assessment of exercise capacity in patients with limited exercise tolerance; the most common include the 6- and 12-minute walk tests (6MWT and 12MWT), the incremental and endurance shuttle walk tests (ISWT and ESWT), incremental and endurance cycle ergometer tests (ICET and ECET), and incremental treadmill tests (TT) and all are well established for clinical use in areas such as cardiovascular disease. ${ }^{25}$ However, it is currently unknown which of these tests best represents the physiological constraints of the disease. The relationship between exercise test performance and the spirometric measurement forced expiratory volume in 1 second $\left(\mathrm{FEV}_{1}\right)^{26,27,28}$ has been established, ${ }^{29}$ however, other key parameters such as forced vital capacity (FVC) ${ }^{30}$ and inspiratory capacity (IC), ${ }^{31,32}$ as well as downstream manifestations of impaired lung function such as reduced partial pressure of arterial oxygen $\left(\mathrm{PaO}_{2}\right),{ }^{33}$ have not.

This systematic review was conducted to assess the correlation between the main outcomes of exercise tests and the most commonly reported physiological and systemic measures of impaired lung function ( $\mathrm{FEV}_{1}$, FVC, IC and $\mathrm{PaO}_{2}$ ) in patients with COPD.

\section{Methods}

\section{Search Strategy}

Literature searches were conducted using Ovid ${ }^{\circledR}$ (Ovid Technologies Inc., New York, New York), incorporating Ovid MEDLINE ${ }^{\circledR}$ (U.S. National Library of Medicine, Bethesda, Maryland) for the period from 1948 to January 22, 2013, Ovid Embase ${ }^{\mathrm{TM}}$ (Elsevier Inc., Philadelphia, Pennsylvania) from 1974 to January 22, 
2013, and The Cochrane Library (John Wiley \& Sons Ltd, Hoboken, New Jersey) from 1962 to January 22, 2013. Search strings were constructed to identify studies reporting primary data on the outcomes of the following exercise tests in patients with COPD (including emphysema- and bronchitis-specific studies): 6MWT, 12MWT, ISWT, ESWT, ICET, ECET and TT. The full search strings used have been published previously. ${ }^{34}$ An example Embase search string is given in the online supplement Figure S1.

\section{Study Selection}

Study selection followed the Preferred Reporting Items for Systematic Reviews and Meta-Analyses (PRISMA) guidelines for performing a systematic literature review. ${ }^{35}$ One researcher screened each reference for inclusion based on title and abstract, and a second researcher performed a full quality-control check. A third researcher resolved any disputes. All publications that met entry criteria for the review were obtained as full articles and reassessed against the review criteria. Data from the selected studies were subsequently used to populate predefined summary tables. All data were fully checked by a second analyst. The review criteria are shown in Table 1. Publications were initially screened based on titles and abstracts, and full articles were reviewed when their relevance was unclear from the abstract. Publications were excluded if they were review articles, were not in English, studied patients with confounding comorbidities (e.g., cancers or diabetes), were unclear on the precise variables used for regression analysis or examined an inappropriate intervention (e.g., non-bronchodilatory pharmacotherapy or homeopathy). Studies were subsequently included for assessment only if they reported data on the correlations (Pearson's $r[r]$ and/or Spearman's rho $[\rho]$ ) between the outcomes of any of the pre-specified exercise tests and the physiological measures of interest: $\mathrm{FEV}_{1}, \mathrm{FVC}, \mathrm{IC}$ and $\mathrm{PaO}_{2}$.

\section{Table 1. Summary of Systematic Review Inclusion/Exclusion Criteria}

\begin{tabular}{|c|c|c|}
\hline & Inclusion & Exclusion \\
\hline Patient Population & Adult patients with COPD & $\begin{array}{l}\text { - Pediatric populations } \\
\text { - Patients with suspected/non-confirmed COPD } \\
\text { - Animal/in vitro studies } \\
\text { - Patients with significant compounding comorbidities } \\
\text { (e.g., cancer or diabetes) }\end{array}$ \\
\hline Interventions & $\begin{array}{l}\text { All forms of approved bronchodilatory } \\
\text { pharmacotherapy or no active therapy }\end{array}$ & Unapproved or experimental therapy (e.g., homeopathy) \\
\hline Outcomes & $\begin{array}{l}\text { Correlation (Pearson's } \mathrm{r}[r] \text { and / or } \\
\text { Spearman's rho }[\rho] \text { ) between } 1 \text { of the } \\
\text { following: } \\
\text { - } 6 \mathrm{MWT} \\
\text { - } 12 \mathrm{MWT} \\
\text { - ISWT } \\
\text { - ESWT } \\
\text { - ICET } \\
\text { - ECET } \\
\text { - TT } \\
\text { AND one of the following: } \\
\text { - FEV } \\
\text { - FVC } \\
\text { - IC } \\
\text { - } \mathrm{PaO}_{2}\end{array}$ & $\begin{array}{l}\text { - Interventional outcomes } \\
\text { - Multivariate analysis results } \\
\text { - Other forms of correlation (including partial correlation } \\
\text { analyses) }\end{array}$ \\
\hline
\end{tabular}

\begin{tabular}{l|l|l}
\hline Study Design & $\begin{array}{l}\text { Prospective or retrospective clinical } \\
\text { trial or observational study }\end{array}$ & Reviews/editorials \\
\hline Publication & English language & Non-English language \\
\hline
\end{tabular}

$\mathrm{COPD}=$ chronic obstructive pulmonary disease; ECET=endurance cycle ergometer test; ESWT=endurance shuttle walk test;

$\mathrm{FEV}_{1}=$ forced expiratory volume in 1 second; $\mathrm{FVC}=$ forced vital capacity; IC=inspiratory capacity; ICET=incremental cycle ergometer test; ISWT=incremental shuttle walk test; $6 \mathrm{MWT}=6$-minute walk test; $12 \mathrm{MWT}=12$ minute walk test; $\mathrm{PaO}_{2}=$ partial pressure of arterial oxygen; TT $=$ treadmill test. 


\section{Data Abstraction}

Data were primarily abstracted by a single author (M.B.) and reviewed by all co-authors. A randomly generated selection of $30 \%$ of all articles was reviewed by a second author (I.F.) for quality control purposes. Extracted study characteristics were: 1)Study objectives (prospective/ retrospective); 2)Study inclusion/exclusion criteria; 3) Study population size; 4)Population baseline characteristics (age, gender, body mass index [BMI], disease severity [staging method and score] and pulmonary function); 5)Methodological information (ECM [protocol, period] and univariant analysis); 6)Results: Pre-test physiological measures, $\mathrm{PaO}_{2}$, arterial oxygen saturation $\left(\mathrm{SaO}_{2}\right)(\%), \mathrm{FEV}_{1}$ (\%), FVC (\%), FEV 1 (L), FVC (L), IC (L), IC (\% pred), functional residual capacity $[\mathrm{FRC}]$ (\%pred), total lung capacity (TLC) (L), TLC (\% pred), residual volume in liters (RV) (L), RV (\% pred), IC/TLC (\%), measure of strength, strength, measure of physical activity, physical activity; 7) Results: Peak physiological measures during test, arterial oxygen saturation $\left(\mathrm{SpO}_{2}\right)$, oxygen consumption $\left(\mathrm{VO}_{2}\right), \mathrm{VO}_{2} / \mathrm{kg}$, heart rate (HR); 8) Results: Patient reported outcomes, exertion (Borg scale), measure of dyspnea, dyspnea, measure of health-related quality of life (HRQoL), HRQoL; 9) Results: demographics, age, sex, height, weight, BMI; 10) Multivariate analyses to explain variance in ECM (parameters, analysis, $\mathrm{r}^{2}$ ); 11) Discussion (conclusions, limitation, comment).

The following outcomes of exercise tests were recorded: distance or stages achieved for the 6MWT, 12MWT, ISWT; duration of exercise for the ESWT and ECET; and the highest recorded volume of oxygen consumption (peak $\mathrm{VO}_{2}$ ) and maximum workload $\left(\mathrm{W}_{\max }\right.$ ) for the TT and ICET. Publications involving studies assessing multivariate regressions were not included owing to the multifactorial nature of the statistical approach and the unsuitability of the output for aggregation.

\section{Statistical Analysis}

Pearson's and Spearman's correlations between lung function test results and the most commonly reported exercise test outcomes are presented. Pearson's correlations are often used to describe the linear association between 2 variables when comparing continuous variable data. Spearman's correlations are commonly used to describe the linear association between 2 sets of ranked (ordinal) data. Correlations are presented as the range of significant values reported in the study publications reviewed. Only those correlations deemed to have achieved significance by the authors of the original articles were included in our descriptive data analysis (i.e., $p<0.05$ ). However, all correlation statistics generated, regardless of significance were extracted (when available, many studies did not provide $r$ values for non-significant correlations). The strength of (significant) correlations is classified according to British Medical Journal guidelines, which regard significant correlation coefficients of $0.0-0.19$ as very weak, $0.20-0.39$ as weak, $0.40-0.59$ as moderate, $0.60-0.79$ as strong and $0.80-1.00$ as very strong correlations.

\section{Inc/usion/Exc/usion Criteria}

Owing to a lack of high quality evidence for associations between these tests and our stated measures of lung function, we included observational studies in our final analysis in addition to randomized controlled trials. Within the results of this search, we reviewed articles to identify those presenting Pearson's and Spearman's correlations between FEV,$F V C$, IC and $\mathrm{PaO}_{2}$, and the most commonly reported exercise test outcomes (described above). Studies reporting lung function variables only as a percentage of age-, sex- and BMIpredicted values were excluded from these results. Publications involving studies assessing multivariate regressions were not included owing to the multifactorial nature of the statistical approach and the unsuitability of the output for aggregation.

\section{Results}

\section{Overview of Identified Studies}

The PRISMA-compliant search methodology used to identify relevant articles is summarized in Figure 1. Of 1781 articles screened, 45 studies were ultimately deemed eligible for inclusion in this review. Table 2 provides a summary of included studies.

\section{Correlations Between Exercise Test Outcomes and FEV}

A total of 39 studies $16,36-65,66-71,72,73$ reported significant Pearson's correlations between an exercise test outcome and baseline $\mathrm{FEV}_{1}$ (Table 3 ). The ranges of correlations reported by studies are presented in Figure 2.

\section{$\mathrm{FEV}_{1}$ and $6 \mathrm{MWT}$}

The most commonly reported test was the 6MWT; of 17 studies $16,36,37,39-41,43,44,46,47,52,54,56,62,65,66,72$ 


\section{Figure 1. PRISMA-compliant Screening and Identification Process}

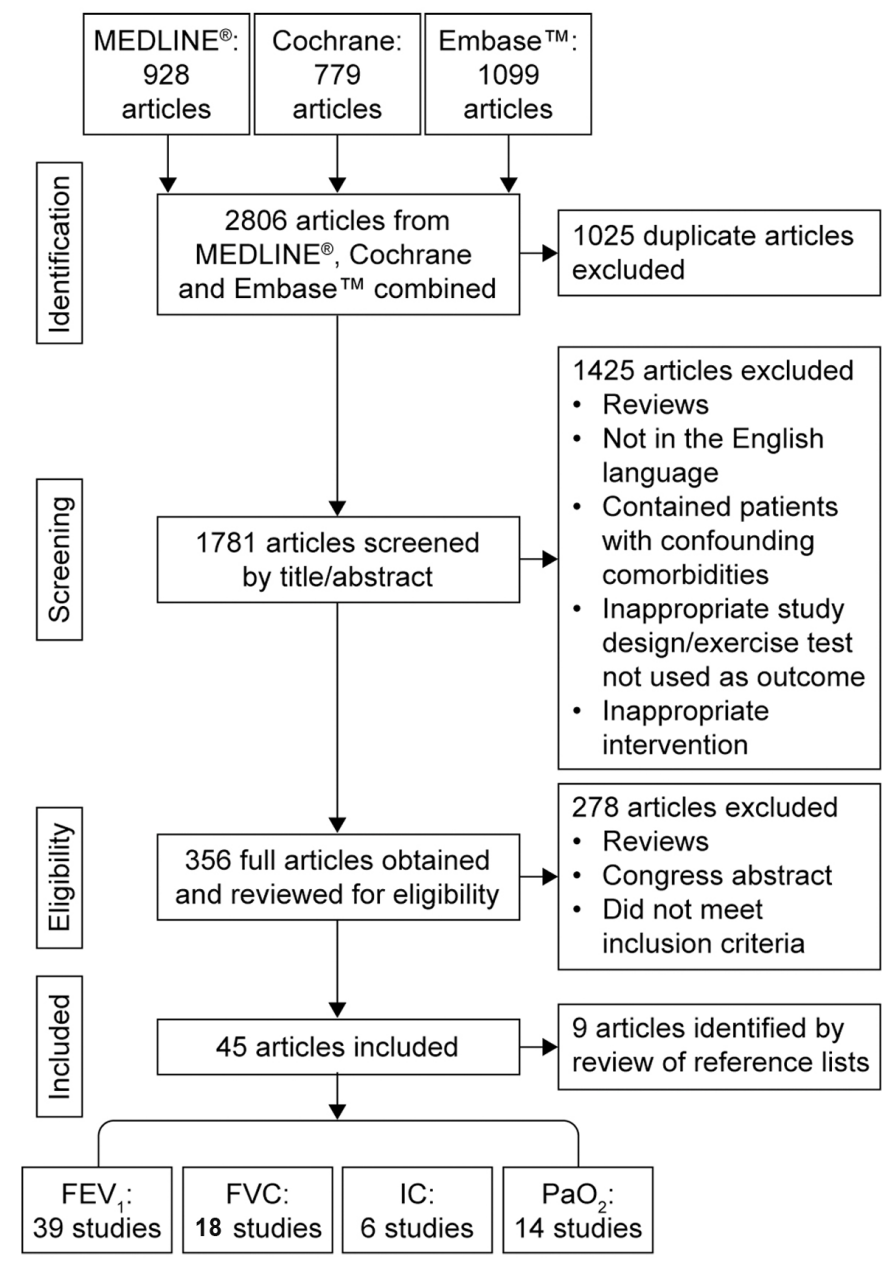

$\mathrm{FEV}_{1}=$ forced expiratory volume in 1 second; $\mathrm{FVC}=$ forced vital capacity; $\mathrm{IC}=$ inspiratory capacity;

$\mathrm{PaO}_{2}=$ partial pressure of arterial oxygen

assessing Pearson's correlations, 12 studies $16,36,39-41,43,47,52,62,65,66,72$ showed significant correlations (weak to strong; $r=0.23-0.62$ ) and 5 reported no statistically significant Pearson's correlation between the 6MWT and $\mathrm{FEV}_{1}$. $37,44,46,54,56$ Additionally, 2 studies 42,63 out of 3 studies $42,63,73$ assessing Spearman's correlations between $\mathrm{FEV}_{1}$ and $6 \mathrm{MWT}$ reported significant correlations (moderate; $\rho=0.41-0.44)$, with the remaining study ${ }^{73}$ reporting no correlation.

\section{$\mathrm{FEV}_{1}$ and $12 \mathrm{MWT}$}

The 12MWT was also reported frequently, with 5 studies $^{38,48,58,60,68}$ out of 8 studies ${ }^{38,48,58-61,64,68}$ reporting significant Pearson's correlations between distance achieved and baseline $\mathrm{FEV}_{1}$ (very weak to strong; $r=0.15-0.62$ ); the remaining 3 studies reported no significant Pearson's correlations. ${ }^{59,61,64}$ One study reported significant correlations between ISWT and $\mathrm{FEV}_{1}$ (weak to moderate; $r=0.22-0.51$ ). ${ }^{69}$

\section{$\mathrm{FEV}_{1}$ and ICET}

The strongest relationship between $\mathrm{FEV}_{1}$ and exercise tests was in studies reporting ICET correlations; all 7 studies $45,47,49,54,59,62,71$ assessing Pearson's correlations between peak $\mathrm{VO}_{2}$ achieved during ICET reported significant correlations (moderate to very strong; $r=0.42-0.83$ ); in 5 studies in which $W_{\max }$ was assessed as the ICET outcome, correlations were also significant (weak to very strong; $r=0.34-0.81) .^{41,55,57,71,72}$ These observations were supported by 1 further study, which reported positive Spearman's correlations for peak $\mathrm{VO}_{2}$ and $\mathrm{W}_{\max }$ during the ICET ( $\rho=0.37$ and 0.55 , respectively). ${ }^{63}$

\section{$\mathrm{FEV}_{1}$ and ISWT and TT}

Limited evidence was available for other test outcomes and baseline $\mathrm{FEV}_{1} ; 1$ study each reported significant Pearson's correlations between FEV 1 and ISWT (weak to moderate; $r=0.22-0.51)^{69}$ and TT (moderate; $r=0.47$, $p<0.05)^{70}$ (Table 3; Figure 2a).

\section{Correlations Between Exercise Test Outcomes and FVC}

Baseline FVC and exercise test outcomes were reported by 18 studies (Table 3; Figure 2b). ${ }^{38,39,41-46,49,50,53,58-62,70}$

\section{FVC and 6MWT}

Again, the most commonly reported Pearson's correlations were between the 6MWT and FVC; 4 studies $39,41,43,62$ out of 6 studies $39,41,43,44,46,62$ assessing Pearson's correlations reported significant correlations (weak to moderate; $r=0.32-0.59$ ), with the remaining 2 studies 44,46 reporting no significant Pearson's correlation. One further study reported a significant Spearman's correlation (moderate; $\rho=0.54$ ). ${ }^{42}$

\section{FVC and 12MWT}

The 12MWT was also assessed frequently, with 4 studies $^{38,58-60}$ of 6 studies ${ }^{38,58-61,64}$ reporting 


\section{Table 2. Summary of Included Studies}

\begin{tabular}{|c|c|c|c|c|c|c|}
\hline Author & Country & $\mathbf{N}$ & Inclusion \& Exclusion Criteria & $\begin{array}{l}\text { Age (years), } \\
\text { Sex }(\mathrm{n}) \\
\text { BMI }\left(\mathrm{kg} / \mathrm{m}^{2}\right)\end{array}$ & Disease Severity & $\begin{array}{l}\text { Pulmonary } \\
\text { Function }\end{array}$ \\
\hline $\begin{array}{l}\text { McGavin } \\
1976^{59}\end{array}$ & $\begin{array}{l}\text { United } \\
\text { Kingdom }\end{array}$ & 35 & $\begin{array}{l}\text { - Inclusion = Patients with chronic } \\
\text { bronchitis } \\
\text { - Exclusion = Not stated }\end{array}$ & $\begin{array}{l}\text { - Age }=40-70 \\
\text { - Male }=35 \\
\text { Female }=0 \\
\text { - } \text { BMI = Not stated }\end{array}$ & $\begin{array}{l}\text { Chronic bronchitis } \\
\text { according to MRC } \\
\text { criteria }\end{array}$ & $\begin{array}{l}-\mathrm{FEV}_{1} \\
\mathrm{~L}=1.05 \pm 0.58 \\
\text { - } \mathrm{FVC} \\
\mathrm{L}=2.84 \pm 0.93\end{array}$ \\
\hline $\begin{array}{l}\text { Mungall } \\
197961\end{array}$ & $\begin{array}{l}\text { United } \\
\text { Kingdom }\end{array}$ & 13 & $\begin{array}{l}\text { - Inclusion = Patients with chronic } \\
\text { bronchitis or radiological emphysema with } \\
\text { no evidence of ischemic heart disease or } \\
\text { other serious illness or reversible } \\
\text { bronchoconstriction } \\
\text { - Exclusion = Not stated }\end{array}$ & $\begin{array}{l}\cdot \text { Age }=55.4 \\
\text { (47-64 range) } \\
\text { - Male }=13, \\
\text { Female }=0 \\
\cdot \text { BMI = Not stated }\end{array}$ & $\begin{array}{l}\text { Chronic bronchitis } \\
\text { according to MRC } \\
\text { criteria or } \\
\text { radiological } \\
\text { emphysema }\end{array}$ & $\begin{array}{l}\text { [Individually } \\
\text { listed in paper] }\end{array}$ \\
\hline $\begin{array}{l}\text { O'Reilly } \\
1982^{64}\end{array}$ & $\begin{array}{l}\text { United } \\
\text { Kingdom }\end{array}$ & 10 & $\begin{array}{l}\text { - Inclusion = Males with chronic airways } \\
\text { obstruction } \\
\text { - Exclusion = Not stated }\end{array}$ & $\begin{array}{l}\cdot \text { Age }=61 \\
(52-70 \text { range) } \\
\cdot \text { Male }=10 \\
\text { Female }=0 \\
\cdot \text { BMI = Not stated }\end{array}$ & $\begin{array}{l}\text { - Chronic } \\
\text { bronchitis }(\mathrm{n}=8) \\
\text { - Radiological } \\
\text { features of } \\
\text { emphysema } \\
(\mathrm{n}=2)\end{array}$ & $\begin{array}{l}-\mathrm{FEV}_{1} \\
\mathrm{~L}=0.81 \pm 0.21 \\
\cdot \mathrm{FVC} \\
\mathrm{L}=2.560 \pm 0.593\end{array}$ \\
\hline $\begin{array}{l}\text { Morgan } \\
1983^{60}\end{array}$ & $\begin{array}{l}\text { United } \\
\text { Kingdom }\end{array}$ & 50 & $\begin{array}{l}\text { - Inclusion = Patients with chronic } \\
\text { bronchitis from several respiratory clinics, } \\
\text { not referred on account of disproportionate } \\
\text { dyspnea, no history of psychiatric illness, } \\
\text { and not taking antidepressant or anxiolytic } \\
\text { drugs } \\
\text { - Exclusion = Patients with any other } \\
\text { disorders that might limit exercise } \\
\text { tolerance }\end{array}$ & $\begin{array}{l}- \text { Age }=60.5 \pm 6.8 \\
\text { - Male }=38 \\
\text { Female }=12 \\
\text { - } \text { BMI = Not stated } \\
\end{array}$ & $\begin{array}{l}\text { Chronic bronchitis } \\
\text { according to MRC } \\
\text { criteria and with } \\
\text { MRC grade 3-5 } \\
\text { dyspnea }\end{array}$ & $\begin{array}{l}-\mathrm{FEV}_{1} \\
\mathrm{~L}=0.97 \pm 0.6 \\
\text { - FVC, } \\
\mathrm{L}=2.59 \pm 0.96\end{array}$ \\
\hline $\begin{array}{l}\text { Light } \\
1985^{58}\end{array}$ & $\begin{array}{l}\text { United } \\
\text { States }\end{array}$ & 45 & $\begin{array}{l}\text { - Inclusion = Patients with COPD, } \mathrm{FEV}_{1} \\
<1.25 \mathrm{~L}, \mathrm{FEV} / \mathrm{FVC}<50 \%, 40-70 \text { years } \\
\text { old, dyspnea-limited exercise tolerance } \\
\text { - Exclusion = Patients who had received } \\
\text { tricyclic antidepressants or other major } \\
\text { antipsychotic drugs in preceding } 2 \text { months, } \\
\text { or who had other significant complicating } \\
\text { diseases such as uncontrolled malignancy, } \\
\text { hepatic insufficiency, insulin-dependent } \\
\text { diabetes mellitus, angina pectoris, } \\
\text { myocardial infarction within previous year, } \\
\text { cardiac arrhythmias, neuromuscular } \\
\text { disease limiting ambulation, or blindness }\end{array}$ & $\begin{array}{l}\text { - Age }=62.4 \pm 4.3 \\
\text { - Male }=45 \\
\text { Female }=0 \\
\text { - BMI = Not stated } \\
\text {, }\end{array}$ & $\begin{array}{l}\text { "Moderate or } \\
\text { severe COPD" }\end{array}$ & $\begin{array}{l}\text { - } \mathrm{FEV}_{1} \\
\mathrm{~L}=0.908 \pm 0.262 \\
\text { - } \mathrm{FEV}_{1} \\
\% \text { predicted } \\
=29.0 \pm 9.0 \\
\text { - FVC, } \\
\mathrm{L}=2.500 \pm 0.606 \\
\text { - FVC } \\
\% \text { predicted } \\
=62.0 \pm 15.5\end{array}$ \\
\hline $\begin{array}{l}\text { Dekhuyzen } \\
1986^{48}\end{array}$ & $\begin{array}{l}\text { Nether- } \\
\text { lands }\end{array}$ & 50 & $\begin{array}{l}\text { - Inclusion = Patients from outpatient } \\
\text { department, age } 40-65 \text { years, suffering } \\
\text { from emphysema and/or chronic } \\
\text { bronchitis with chronic airflow obstruction, } \\
\text { steady clinical condition for preceding } 2 \\
\text { months, } \mathrm{FEV}_{1} \text { increased by }<20 \% \text { after } \\
\text { inhalation of salbutamol } \\
\text { - Exclusion = Patients with hypertension, } \\
\text { ischemic heart disease, peripheral vascular } \\
\text { disease or muscular disease }\end{array}$ & $\begin{array}{l}- \text { Age }=57.2 \pm 7.3 \\
- \text { Male }=37 \\
\text { Female }=13 \\
\cdot \text { BMI = Not stated }\end{array}$ & $\begin{array}{l}\text { As per MRC } \\
\text { criteria }\end{array}$ & $\begin{array}{l}-\mathrm{FEV}_{1} \\
\mathrm{~L}=1.463 \pm 0.566 \\
\text { - IVC, } \\
\mathrm{L}=3.712 \pm 1.059\end{array}$ \\
\hline
\end{tabular}




\begin{tabular}{|c|c|c|c|c|c|c|}
\hline $\begin{array}{l}\text { Chonan } \\
1988^{78}\end{array}$ & Japan & 15 & $\begin{array}{l}\text { - Inclusion = Patients with clinically stable } \\
\text { chronic emphysema and chronic } \\
\text { bronchitis who had been observed in } \\
\text { pulmonary clinic for > } 1 \text { year, had } \\
\text { undergone repeated pulmonary function } \\
\text { tests demonstrating chronic airway } \\
\text { obstruction and lung hyperinflation, had } \\
\text { no substantial changes in pulmonary } \\
\text { function during preceding } 6 \text { months } \\
\text { - Exclusion = Not stated }\end{array}$ & $\begin{array}{l}\text { - Mean age } \\
=63 \pm 6.5 \\
\text { - Male }=15 \\
\text { Female }=0 \\
\text { - BMI = Not stated }\end{array}$ & $\begin{array}{l}\text { Diagnoses } \\
\text { consistent with } \\
\text { ATS standards }\end{array}$ & $\begin{array}{l}\text { - } \mathrm{FEV}_{1} \\
\% \text { predicted } \\
=36.9 \pm 13.6 \\
\text { - VC, } \\
\% \text { predicted } \\
=74.6 \pm 21.5\end{array}$ \\
\hline $\begin{array}{l}\text { Chetty } \\
1989^{45}\end{array}$ & $\begin{array}{l}\text { United } \\
\text { States }\end{array}$ & 37 & $\begin{array}{l}\text { - Inclusion = Patients with clinically stable } \\
\text { COPD with no heart failure on clinical } \\
\text { examination or symptomatic coronary } \\
\text { artery disease } \\
\text { - Exclusion = Not stated }\end{array}$ & $\begin{array}{l}\text { - Age }=60 \pm 6 \\
\text { - Male } / \text { Female } \\
=\text { Not stated } \\
\text { - BMI = Not stated }\end{array}$ & $\begin{array}{l}\text { Moderate to severe } \\
\text { COPD }\end{array}$ & $\begin{array}{l}\cdot \mathrm{FEV}_{1} \\
\mathrm{~L}=1.49 \pm 0.59 \\
\cdot \mathrm{FEV}_{1} / \mathrm{FVC} \\
\%=48 \pm 12\end{array}$ \\
\hline $\begin{array}{l}\text { Dillard } \\
1989^{49}\end{array}$ & $\begin{array}{l}\text { United } \\
\text { States }\end{array}$ & 20 & $\begin{array}{l}\text { - Inclusion = Patients fulfilling the study } \\
\text { definitions of chronic airflow obstruction } \\
\text { and ventilatory limitation of exercise, the } \\
\text { latter defined by: (1) Peak exercise cardiac } \\
\text { frequency < } 2 \text { SD below predicted } \\
\text { maximum, (2) dyspnea as exercise limiting } \\
\text { symptom, (3) exercise ventilation }>80 \% \\
\text { of } 12 \mathrm{~s} \mathrm{MVV} \\
\text { - Exclusion = Patients who did not meet } \\
\text { the definitions or who had a history of } \\
\text { prior acute or chronic ventilatory failure } \\
\text { or cardiac disease }\end{array}$ & $\begin{array}{l}- \text { Age }=56 \pm 3 \\
\text { - Male }=20 \\
\text { Female }=0 \\
\text { - BMI = Not stated }\end{array}$ & $\begin{array}{l}\text { Chronic airflow } \\
\text { obstruction defined } \\
\text { by: } \\
\text { (1) } \mathrm{FEV}_{1} / \mathrm{FVC} \\
<0.7 \\
\text { (2) TLC } \\
>80 \% \text { predicted } \\
\text { (3) change in } \mathrm{FEV}_{1} \\
<15 \% \text { after } \\
\text { bronchodilation }\end{array}$ & $\begin{array}{l}-\mathrm{FEV}_{1} \\
\mathrm{~L}=1.72 \pm 0.21 \\
\text { - FVC } \\
\mathrm{L}=3.27 \pm 0.26\end{array}$ \\
\hline $\begin{array}{l}\text { Schols } \\
198968\end{array}$ & $\begin{array}{l}\text { Nether- } \\
\text { lands }\end{array}$ & 83 & $\begin{array}{l}\text { - Inclusion = Patients with clinically stable } \\
\text { severe COPD admitted to PR program } \\
\text { - Exclusion = Patients with cardiovascular, } \\
\text { neurological, endocrine and locomotor } \\
\text { diseases and those with } \mathrm{PaO}_{2}<7.3 \mathrm{kPa}\end{array}$ & $\begin{array}{l}\text { - Age }=62 \pm 8 \\
\text { - Male }=71 \\
\text { Female }=12 \\
\text { - BMI = Not stated }\end{array}$ & Severe COPD & $\begin{array}{l}\cdot \mathrm{FEV}_{1} \\
\mathrm{~L}=0.9 \pm 0.3 \\
\cdot \mathrm{IVC} \\
\mathrm{L}=2.7 \pm 0.6\end{array}$ \\
\hline $\begin{array}{l}\text { Singh } \\
1992^{69}\end{array}$ & $\begin{array}{l}\text { United } \\
\text { Kingdom }\end{array}$ & 10 & $\begin{array}{l}\text { - Inclusion = Patients with COPD } \\
\text { recruited from medical clinics } \\
\text { - Exclusion = Patients known to be } \\
\text { hypoxic with cor pulmonale or ischemic } \\
\text { heart disease, participants with } \\
\text { neurological or locomotor disorders }\end{array}$ & $\begin{array}{l}\text { Groups A, B, C: } \\
\cdot \mathrm{n}=10,10,15 \\
\cdot \text { Age }= \\
64 \text { ( } 54-73 \text { range), } \\
63 \text { (52-74 range), } \\
64 \text { (45-71 range) } \\
\text { Male }=9,6,10 \\
\cdot \text { BMI = Not stated }\end{array}$ & NR & $\begin{array}{l}\text { Groups } \mathrm{A}, \mathrm{B}, \mathrm{C}: \\
\cdot \mathrm{FEV}_{1}, \\
\mathrm{~L}=0.50 \\
(0.36-1.45 \text { range)}, \\
1.10 \\
(0.60-2.10 \text { range), } \\
1.20 \\
(0.50-2.85 \text { range) }\end{array}$ \\
\hline $\begin{array}{l}\text { Wakayama } \\
1993^{70}\end{array}$ & Japan & 20 & $\begin{array}{l}\text { - Inclusion = Patients with clinically } \\
\text { stable pulmonary emphysema who } \\
\text { entered a PR program and free of other } \\
\text { diseases, except for mild hypertension } \\
\text { - Exclusion = Patients receiving home } \\
\text { oxygen therapy }\end{array}$ & $\begin{array}{l}\text { - Age }=66 \pm 6 \\
\text { - Male } / \text { Female } \\
=\text { Not stated } \\
\text { - BMI = Not stated }\end{array}$ & Not stated & $\begin{array}{l}\cdot \mathrm{FEV}_{1}, \\
\mathrm{~L}=1.67 \pm 0.50 \\
\cdot \mathrm{FEV}_{1}, \\
\% \text { predicted } \\
=52 \pm 22 \\
\cdot \mathrm{FVC}, \\
\mathrm{L}=2.62 \pm 0.68 \\
-\mathrm{FVC} \\
\% \text { predicted } \\
=80 \pm 19\end{array}$ \\
\hline $\begin{array}{l}\text { Bernstein } \\
1994^{38}\end{array}$ & $\begin{array}{l}\text { United } \\
\text { States }\end{array}$ & 9 & $\begin{array}{l}\text { - Inclusion }=\text { COPD patients with } \mathrm{FEV}_{1} \\
<1.4 \mathrm{~L}, \mathrm{FEV} \mathrm{V}_{1} / \mathrm{FVC}<0.50 \text {, exercise } \\
\text { limited by dyspnea, stable disease state, } \\
\text { and of age } 40-75 \text { years } \\
\text { - Exclusion = Patients with known left } \\
\text { ventricular disease or neuromuscular or } \\
\text { other medical problems which would } \\
\text { preclude them from walking for } 12 \text { min } \\
\text { or performing cycle ergometry }\end{array}$ & $\begin{array}{l}\text { - Age }=67 \pm 4 \\
\text { - Male }=9 \\
\text { Female }=0 \\
\text { - BMI = Not stated }\end{array}$ & $\begin{array}{l}\text { "Average patient } \\
\text { had moderate } \\
\mathrm{COPD}^{\text {" based on }} \\
\text { mean } \mathrm{FEV}_{1}\end{array}$ & $\begin{array}{l}\cdot \text { FVC, } \\
\mathrm{L}=3.40 \pm 0.61 \\
\cdot \mathrm{FEV}_{1} \\
\mathrm{~L}=1.32 \pm 0.28\end{array}$ \\
\hline
\end{tabular}




\begin{tabular}{|c|c|c|c|c|c|c|}
\hline $\begin{array}{l}\text { Wijkstra } \\
1994^{72}\end{array}$ & $\begin{array}{l}\text { Nether- } \\
\text { lands }\end{array}$ & 40 & $\begin{array}{l}\text { - Inclusion = Patients with known COPD } \\
\text { with post-bronchodilator } \mathrm{FEV}_{1}<60 \% \\
\text { predicted and } \mathrm{FEV}_{1} / \mathrm{IVC}<50 \% \text {, and } \\
\text { clinically stable } \\
\text { - Exclusion = Patients with evidence of } \\
\text { ischemic heart disease, intermittent } \\
\text { claudication, musculoskeletal disorders, } \\
\text { or other disabling diseases that might } \\
\text { restrict a pulmonary rehabilitation } \\
\text { program }\end{array}$ & $\begin{array}{l}\text { - Age = } 62.4 \pm 5.0 \\
\text { - Male/Female } \\
=\text { Not stated } \\
\text { - BMI = Not stated }\end{array}$ & $\begin{array}{l}\text { Severe airways } \\
\text { obstruction, based } \\
\text { on pulmonary } \\
\text { function indices }\end{array}$ & $\begin{array}{l}\text { - } \mathrm{FEV}_{1} \\
\mathrm{~L}=1.2 \pm 0.3 \\
\text { - } \mathrm{FEV}_{1} \\
\% \text { predicted } \\
=44.3 \pm 10.6 \\
\text { - IVC, } \\
\mathrm{L}=3.6 \pm 0.9 \\
\text { - IVC, } \\
\% \text { predicted } \\
=91.3 \pm 17.2\end{array}$ \\
\hline $\begin{array}{l}\text { Gosselink } \\
1996^{54}\end{array}$ & Belgium & 41 & $\begin{array}{l}\text { - Inclusion }=\text { Patients with COPD } \\
\text { attending the outpatient clinic for dyspnea } \\
\text { and poor exercise tolerance with: } \mathrm{FEV}_{1} \\
\text { between } 20 \% \text { and } 60 \% \text { of predicted value, } \\
\text { clinically stable condition, < } 75 \text { years old, } \\
\text { no recent cardiac complaints, and no } \\
\text { other pathological conditions } \\
\text { - Exclusion = Not stated }\end{array}$ & $\begin{array}{l}\text { - Age = } 58 \pm 10 \\
\text { - Male = 32, } \\
\text { Female = } 9 \\
\text { - } \mathrm{BMI}=23 \pm 5\end{array}$ & $\begin{array}{l}\text { "Moderate to severe } \\
\text { COPD" }\end{array}$ & $\begin{array}{l}\text { - } \mathrm{FEV}_{1} \text {, } \\
\% \text { predicted } \\
=43 \pm 19 \\
\text { - FVC, } \\
\% \text { predicted } \\
=79 \pm 21\end{array}$ \\
\hline $\begin{array}{l}\text { Baarends } \\
1997^{80}\end{array}$ & $\begin{array}{l}\text { Nether- } \\
\text { lands }\end{array}$ & 62 & $\begin{array}{l}\text { - Inclusion = Patients with COPD admitted } \\
\text { to pulmonary rehabilitation center in } \\
\text { stable clinical condition, who were not } \\
\text { suffering from respiratory tract infection } \\
\text { or clinically visible signs of edema at the } \\
\text { time of study, who did not require } \\
\text { supplemental oxygen, and had no known } \\
\text { cardiovascular, neurological, endocrine or } \\
\text { locomotive diseases } \\
\text { - Exclusion = Patients with an increase in } \\
\text { FEV } V_{1} \text { of }>10 \% \text { baseline after inhalation of } \\
\text { beta2-agonists }\end{array}$ & $\begin{array}{l}- \text { Age }=63 \pm 9 \\
\text { - } \text { Male = 44, } \\
\text { Female = } 18 \\
\cdot \text { BMI }=23.2 \pm 4.8\end{array}$ & $\begin{array}{l}\text { Moderate to severe } \\
\text { COPD, as per ATS } \\
\text { definitions }\end{array}$ & $\begin{array}{l}\text { - } \mathrm{FEV}_{1} \text {, } \\
\% \text { predicted } \\
=39 \pm 13 \\
\text { - FVC, } \\
\% \text { predicted } \\
=87 \pm 16\end{array}$ \\
\hline $\begin{array}{l}\text { Borak } \\
1998^{39}\end{array}$ & Poland & 49 & $\begin{array}{l}\text { - Inclusion = Patients with COPD } \\
\text { diagnosis, based on history, clinical } \\
\text { examination, lung function tests and chest } \\
\text { radiography, in stable state, with no signs } \\
\text { of respiratory infection or heart failure or } \\
\text { any concomitant disease that could } \\
\text { influence their exercise tolerance or their } \\
\text { psychological status. They were treated } \\
\text { with inhaled bronchodilators - ipratropium } \\
\text { bromide, beta2-agonists and oral } \\
\text { theophylline. Six patients were on } \\
\text { domiciliary long-term oxygen therapy } \\
\text { - Exclusion = patients with asthma, > 10\% } \\
\text { improvement in FEV } 1 \text { and/or FVC after } \\
\text { inhalation of beta2-agonists, and blood or } \\
\text { sputum eosinophilia }\end{array}$ & $\begin{array}{l}\text { - Age }=58 \pm 8 \\
\text { - Male }=38, \\
\text { Female }=11 \\
\text { - BMI = Not stated } \\
\end{array}$ & $\begin{array}{l}\text { "wide spectrum } \\
\text { of airway } \\
\text { limitation severity } \\
\text { and hypoxemia" }\end{array}$ & $\begin{array}{l}\text { - FVC, } \\
\mathrm{L}=2.6 \pm 0.7 \\
\text { - FVC, } \\
\% \text { predicted } \\
=60 \pm 16 \\
\text { - FEV } \\
\mathrm{L}=1.0 \pm 0.5 \\
\text { - FEV } \\
\% \text { predicted } \\
=32 \pm 14\end{array}$ \\
\hline $\begin{array}{l}\text { Revill } \\
1999^{67}\end{array}$ & $\begin{array}{l}\text { United } \\
\text { Kingdom }\end{array}$ & 10 & $\begin{array}{l}\text { - Inclusion }=\text { Patients in Group A were } \\
\text { recruited from outpatient respiratory } \\
\text { clinics, patients in Groups B \& C were } \\
\text { recruited from waiting lists for pulmonary } \\
\text { rehabilitation, all patients had COPD, } \\
\text { FEV }_{1}<60 \% \text { predicted, and self-reported } \\
\text { breathlessness due to exercise } \\
\text { - Exclusion = Not stated }\end{array}$ & $\begin{array}{l}\text { - Age = } 63.7 \pm 5.5 \\
\text { - Male = 9, } \\
\text { Female = } 1 \\
\text { - BMI = Not stated }\end{array}$ & $\begin{array}{l}\text { COPD diagnosed } \\
\text { according to BTS } \\
\text { guidelines }\end{array}$ & $\begin{array}{l}-\mathrm{FEV}_{1}, \\
\mathrm{~L}=1.01 \pm 0.36 \\
\cdot \mathrm{FEV}_{1} \\
\% \text { predicted } \\
=35 \pm 4 \\
\text { - FVC, } \\
\mathrm{L}=2.92 \pm 0.55 \\
\text { - FVC, } \\
\% \text { predicted } \\
=81 \pm 20\end{array}$ \\
\hline
\end{tabular}




\begin{tabular}{|c|c|c|c|c|c|c|}
\hline $\begin{array}{l}\text { Yoshikawa } \\
1999^{71}\end{array}$ & Japan & 27 & $\begin{array}{l}\text { - Inclusion = Male patients with stable } \\
\text { COPD, no other pathologic conditions and } \\
\text { not receiving oral corticosteroids } \\
\text { - Exclusion = Not stated }\end{array}$ & $\begin{array}{l}\text { - } \text { Age }=68.4 \pm 5.8 \\
\text { - Male }=27 \\
\text { Female }=0 \\
\text { - } \text { BMI }=18.4 \pm 2.3\end{array}$ & COPD as per ATS & $\begin{array}{l}\cdot \mathrm{FEV}_{1} \\
\mathrm{~L}=1.18 \pm 0.66 \\
\cdot \mathrm{FEV}_{1}, \\
\% \text { predicted } \\
=49.8 \pm 26.4 \\
\cdot \mathrm{VC}, \\
\mathrm{L}=2.75 \pm 0.83 \\
\cdot \mathrm{VC}, \\
\% \text { predicted } \\
=84.0 \pm 23.5\end{array}$ \\
\hline $\begin{array}{l}\text { Rejeski } \\
2000^{66}\end{array}$ & $\begin{array}{l}\text { United } \\
\text { States }\end{array}$ & 209 & $\begin{array}{l}\text { - Inclusion = Diagnosis of COPD, } 55-80 \\
\text { years old, self-reported disability attributed } \\
\text { to breathlessness when performing daily } \\
\text { activities, prior or current history of } \\
\text { smoking, FEV }_{1} / \mathrm{FVC} \text { ratio } \leq 70 \% \text { and } \\
\text { FEV }_{1}>20 \% \text { of predicted. } \\
\text { - Exclusion = Not stated }\end{array}$ & $\begin{array}{l}\text { - Age }=67.2 \pm 6.0 \\
\text { - Male }=117 \\
\text { Female }=92 \\
\text { - BMI = Not stated }\end{array}$ & $\begin{array}{l}\mathrm{n} \text { in ATS stage of } \\
\text { COPD: } \\
\text { - Stage I mild: } 134 \\
\text { - Stage II moderate: } \\
55 \\
\text { - Stage III severe: } \\
20\end{array}$ & $\begin{array}{l}\text { - } \mathrm{FEV}_{1}, \\
\mathrm{~L}=1.57 \pm 0.58 \\
\text { - } \mathrm{FEV}_{1} \\
\% \text { predicted } \\
=57.10 \pm 17.00\end{array}$ \\
\hline $\begin{array}{l}\text { Chuang } \\
2001^{46}\end{array}$ & $\begin{array}{l}\text { United } \\
\text { States/ } \\
\text { Taiwan }\end{array}$ & 27 & $\begin{array}{l}\text { - Inclusion }=\text { Outpatients with clinically } \\
\text { stable COPD, receiving a regular schedule } \\
\text { of administered bronchodilators with or } \\
\text { without oral prednisolone of }<10 \mathrm{mg} / \text { day, } \\
\text { who had peak exercise heart rate } \geq 85 \% \text { of } \\
\text { the maximally predicted, RER } \geq 1.09 \text { at } \\
\text { peak exercise, arterial plasma bicarbonate } \\
\text { at peak exercise decreases from resting } \\
\text { baseline level by at least } 4 \text { mmol/L, plasma } \\
\text { pH value at peak exercise } \leq 7.35 \\
\text { - Exclusion = Patients with significant } \\
\text { arrhythmia or having a history of } \\
\text { malignancy, cardiovascular or peripheral } \\
\text { vascular disease, or locomotion problems }\end{array}$ & $\begin{array}{l}\text { - Age }=65 \pm 6 \\
\text { - Male }=27 \\
\text { Female }=0 \\
\text { - BMI = Not stated } \\
\end{array}$ & $\begin{array}{l}\text { Moderate to severe } \\
\text { COPD, based on } \\
\text { most ( 90\%) } \\
\text { patients having } \\
\mathrm{FEV}_{1} / \mathrm{VC}<65 \%\end{array}$ & $\begin{array}{l}-\mathrm{FEV}_{1} \\
\mathrm{~L}=1.2 \pm 0.4 \\
\cdot \mathrm{FEV}_{1} \\
\% \text { predicted } \\
=49 \pm 10 \\
\cdot \mathrm{VC} \\
\mathrm{L}=2.8 \pm 0.5\end{array}$ \\
\hline $\begin{array}{l}\text { Dowson } \\
2001^{50}\end{array}$ & $\begin{array}{l}\text { United } \\
\text { Kingdom }\end{array}$ & 29 & $\begin{array}{l}\text { - Inclusion = Patients with a1-antitrypsin } \\
\text { deficiency and macroscopic emphysema } \\
\text { selected consecutively from a treatment } \\
\text { center } \\
\text { - Exclusion = Patients with asthma, } \\
\text { bronchiectasis, liver disease or other } \\
\text { medical problems likely to limit exercise } \\
\text { or alter health status }\end{array}$ & $\begin{array}{l}\text { - Median age } \\
=52(\mathrm{IQR}: 46-60) \\
\text { - Male = } 19, \\
\text { Female = } 10 \\
\text { - } \text { BMI = Not stated }\end{array}$ & $\begin{array}{l}\text { Moderate to severe } \\
\text { airflow obstruction }\end{array}$ & $\begin{array}{l}\text { - Median FEV } \\
\text { L = } 1.03 \\
\text { (IQR: } 0.84-1.41 \text { ) } \\
\text { - Median } \mathrm{FEV}_{1} \text {, } \\
\text { \% predicted = } 35 \\
\text { - Median } \mathrm{FEV}_{1} / \\
\text { VC, L = } 0.31 \\
\text { (IQR: } 0.25-0.43 \text { ) } \\
\text { - Median FEV } / \\
\text { VC, \% predicted } \\
=37\end{array}$ \\
\hline $\begin{array}{l}\text { Fujita } \\
2002^{53}\end{array}$ & Japan & 20 & $\begin{array}{l}\text { - Inclusion = Patients with chronic } \\
\text { stable emphysema } \\
\text { - Exclusion = Not stated }\end{array}$ & $\begin{array}{l}\text { - Age }=66.9 \pm 8.6 \\
\text { - Male }=18 \\
\text { Female }=2 \\
\text { - BMI = Not stated }\end{array}$ & Not stated & $\begin{array}{l}\text { - } \mathrm{FEV}_{1} \\
\mathrm{~L}=1.31 \pm 0.61 \\
\text { - } \mathrm{FEV}_{1} \\
\% \text { predicted } \\
=44.4 \pm 19.8 \\
\text { - } \mathrm{VC} \\
\mathrm{L}=3.1 \pm 0.8 \\
\text { - VC, } \\
\% \text { predicted } \\
=94.7 \pm 17.8\end{array}$ \\
\hline
\end{tabular}




\begin{tabular}{|c|c|c|c|c|c|c|}
\hline $\begin{array}{l}\text { Oga } \\
2002^{63}\end{array}$ & Japan & 36 & $\begin{array}{l}\text { - Inclusion }=\text { Consecutive male patients } \\
\text { with stable COPD from placebo arm of } \\
\text { previously reported clinical trial with age } \\
>45 \text { years, smoking history }>20 \text { pack } \\
\text { years, chest radiographs showing } \\
\text { hyperinflation, } \mathrm{FEV}_{1} \text { predicted }<80 \% \text {, } \\
\text { post-bronchodilator } \mathrm{FEV}_{1} / \mathrm{FVC}<0.7 \\
\text { Exclusion = Patients with exacerbations } \\
\text { in preceding } 3 \text { months, history of asthma, } \\
\text { other diseases likely to affect exercise, or } \\
\text { hypoxemia at rest }\end{array}$ & $\begin{array}{l}- \text { Age }=69 \pm 7 \\
\text { - } \text { Male }=36 \\
\text { Female }=0 \\
\cdot \text { BMI = 20.3 } \pm 3.2\end{array}$ & COPD as per ATS & $\begin{array}{l}\text { - } \mathrm{FEV}_{1} \\
\mathrm{~L}=1.07 \pm 0.45 \\
\text { - } \mathrm{FEV}_{1}, \\
\% \text { predicted } \\
=40.3 \pm 16.7\end{array}$ \\
\hline $\begin{array}{l}\text { Carter } \\
2003^{43}\end{array}$ & $\begin{array}{l}\text { United } \\
\text { States }\end{array}$ & 124 & $\begin{array}{l}\text { - Inclusion = Individuals with moderate to } \\
\text { very severe COPD, capable of undergoing } \\
\text { exercise testing to peak effort, greater than } \\
\text { 8th grade education and able to read, no } \\
\text { interfering coexisting medical conditions } \\
\text { - Exclusion = cardiac, renal or endocrine } \\
\text { disease, claudication limiting exercise } \\
\text { capacity, musculoskeletal pain, syncope, } \\
\text { significant ST-T depression or cardiac } \\
\text { arrhythmia on exercise testing, or a } \\
\text { pattern for restrictive lung disease }\end{array}$ & $\begin{array}{l}\text { - Age }=66.8 \pm 7.3 \\
\text { - } \text { Male =90, } \\
\text { Female = } 34 \\
\cdot \text { BMI = } 27.1 \pm 5.2\end{array}$ & $\begin{array}{l}\text { Moderate to severe } \\
\text { airway obstruction } \\
\text { as determined by } \\
\text { pulmonary function } \\
\text { indices }\end{array}$ & $\begin{array}{l}-\mathrm{FEV}_{1} \\
\mathrm{~L}=1.33 \pm 0.43 \\
-\mathrm{FEV}_{1} \\
\% \text { predicted } \\
=45.9 \pm 12.5 \\
\text { - } \mathrm{FVC}, \\
\mathrm{L}=3.25 \pm 0.91 \\
\text { - FVC, } \\
\% \text { predicted } \\
=82.9 \pm 15.5\end{array}$ \\
\hline $\begin{array}{l}\text { Hodgev } \\
2003^{56}\end{array}$ & Bulgaria & 20 & $\begin{array}{l}\text { - Inclusion = Patients with clinically stable } \\
\text { COPD who had not received systemic } \\
\text { steroids at least } 2 \text { months prior to study, } \\
\text { but did receive therapy with } \\
\text { bronchodilators during study } \\
\text { - Exclusion = Patients with history of } \\
\text { asthma, allergic rhinitis or atrophy; active } \\
\text { lung tuberculosis or lung carcinoma; } \\
\text { cardiovascular disorders including } \\
\text { myocardial infarction, angina pectoris, } \\
\text { pericarditis, valvular diseases (excepting } \\
\text { relative tricuspid insufficiency), } \\
\text { arrhythmia, arterial hypertension requiring } \\
\text { drug treatment; disorders of locomotor } \\
\text { apparatus; anemias; kidney, liver or } \\
\text { metabolic disorders }\end{array}$ & $\begin{array}{l}\text { - Age }=55.9 \pm 8.7 \\
\text { - Male }=20 \\
\text { Female }=0 \\
\text { - BMI = } 27.8 \pm 7.7\end{array}$ & $\begin{array}{l}\text { COPD diagnosed as } \\
\text { per guidelines } \\
\text { recommended by } \\
\text { the National } \\
\text { Consensus } \\
\text { Conference }\end{array}$ & $\begin{array}{l}\cdot \mathrm{FEV}_{1}, \\
\mathrm{~L}=1.35 \pm 0.72 \\
\cdot \mathrm{FEV}_{1} \\
\% \text { predicted } \\
=42 \pm 19\end{array}$ \\
\hline $\begin{array}{l}\text { Peruzza } \\
2003^{65}\end{array}$ & Italy & 60 & $\begin{array}{l}\text { - Inclusion }=\text { Patients with COPD over the } \\
\text { age of } 65 \text { years } \\
\text { - Exclusion }=\text { Patients who were } \\
\text { underweight }\left(\mathrm{BMI}<18.5 \mathrm{~kg} / \mathrm{m}^{2}\right) \text { or obese } \\
\left(\mathrm{BMI}>30 \mathrm{~kg} / \mathrm{m}^{2}\right) \text {, had ischemic heart } \\
\text { disease, or experienced changes in } \\
\text { medication in preceding } 30 \text { days or } \\
\text { hospital admission in preceding } 6 \text { weeks }\end{array}$ & $\begin{array}{l}- \text { Age }=74.5 \pm 5.8 \\
\text { - Male }=60 \\
\text { Female }=0 \\
\cdot \text { BMI = 25.1 } \pm 3.8\end{array}$ & As per ERS criteria & $\begin{array}{l}-\mathrm{FEV}_{1} \\
\mathrm{~L}=1.1 \pm 0.5 \\
\text { - } \mathrm{FEV}_{1} \\
\% \text { predicted } \\
=48.1 \pm 18.3\end{array}$ \\
\hline $\begin{array}{l}\text { Vagaggini } \\
2003^{76}\end{array}$ & Italy & 18 & $\begin{array}{l}\text { - Inclusion }=\text { Patients who were in the } \\
\text { recovery phase of an exacerbation of COPD } \\
\text { that had required hospitalization, had } \\
\text { never performed a 6MWT or ISWT, were } \\
\text { taking bronchodilators and/or inhaled } \\
\text { corticosteroids at the time of study, and } \\
\text { were examined at least } 14 \text { days after the } \\
\text { beginning of the exacerbation } \\
\text { - Exclusion = Not stated }\end{array}$ & $\begin{array}{l}\text { - Age }=67 \pm 8.2 \\
\text { - Male = } 15 \\
\text { Female }=3 \\
\text { - BMI = Not stated }\end{array}$ & $\begin{array}{l}\text { Moderate to severe } \\
\text { COPD }\end{array}$ & $\begin{array}{l}\text { - } \mathrm{FEV}_{1} \text {, } \\
\% \text { predicted } \\
=48 \pm 14 \\
\text { - FVC, } \\
\% \text { predicted } \\
=74.4 \pm 16.2\end{array}$ \\
\hline
\end{tabular}




\begin{tabular}{|c|c|c|c|c|c|c|}
\hline $\begin{array}{l}\text { Nakamura } \\
2004^{62}\end{array}$ & Japan & 38 & $\begin{array}{l}\text { - Inclusion }=\text { Male patients with clinically } \\
\text { stable COPD and } \mathrm{FEV}_{1}<70 \% \text { predicted, } \\
\text { age }<80 \text { years, and no other pathological } \\
\text { conditions } \\
\text { - Exclusion }=\text { Not stated }\end{array}$ & $\begin{array}{l}\text { - Age }=69.8 \pm 6.7 \\
\text { - Male }=38 \\
\text { Female }=0 \\
\text { - BMI }=21.4 \pm 3.1\end{array}$ & $\begin{array}{l}\text { "Moderate to severe } \\
\text { COPD" }\end{array}$ & $\begin{array}{l}-\mathrm{FEV}_{1} \\
\mathrm{~L}=1.33 \pm 0.55 \\
\text { - } \mathrm{FEV}_{1} \\
\% \text { predicted } \\
=49.5 \pm 19.6 \\
\text { - FVC, } \\
\mathrm{L}=2.79 \pm 0.70\end{array}$ \\
\hline $\begin{array}{l}\text { Pinto-Plata } \\
2004^{16}\end{array}$ & $\begin{array}{l}\text { United } \\
\text { States }\end{array}$ & 198 & $\begin{array}{l}\text { - Inclusion = Patients with COPD referred } \\
\text { to hospital pulmonary department } \\
\text { - Exclusion = Patients who had had an } \\
\text { exacerbation in preceding } 4 \text { months or } \\
\text { another unstable medical problem }\end{array}$ & $\begin{array}{l}- \text { Age }=68 \pm 9 \\
- \text { Male }=168 \\
\text { Female }=30 \\
\text { - } \mathrm{BMI}=24.86 \pm \\
5.74\end{array}$ & COPD as per ATS & $\begin{array}{l}\cdot \mathrm{FEV}_{1} \\
\mathrm{~L}=1.04 \pm 0.39\end{array}$ \\
\hline $\begin{array}{l}\text { Behnke } \\
2005^{37}\end{array}$ & Germany & 88 & $\begin{array}{l}\text { - Inclusion = Individuals with COPD and } \\
\mathrm{FEV}_{1} \leq 75 \% \text { predicted and no other } \\
\text { significant airway disease } \\
\text { - Exclusion = Not stated }\end{array}$ & $\begin{array}{l}\text { Training group } \\
(\mathrm{n}=66): \\
\text { - Age }=61.2 \pm 8.6 \\
\text { - Male }=51, \\
\text { Female }=15 \\
\text { - } \text { BMI }=24.5 \pm 3.0 \\
\text { Control group } \\
(\mathrm{n}=22): \\
\text { - Age }=58.4 \pm 6.7 \\
\text { - Male }=20, \\
\text { Female }=2 \\
\text { - BMI }=23.8 \pm 4.9\end{array}$ & $\begin{array}{l}\text { Mild to severe COPD } \\
\text { diagnosis as per } \\
\text { international } \\
\text { guidelines (GOLD) }\end{array}$ & $\begin{array}{l}\text { Training group, } \\
\text { control group: } \\
\text { - } \mathrm{FEV}_{1} \text {, } \\
\mathrm{L}=1.30 \pm 0.49 \\
1.45 \pm 0.51 \\
\text { - } \mathrm{FEV}_{1} \\
\% \text { predicted } \\
=41.9 \pm 13.9 \\
46.9 \pm 15.1\end{array}$ \\
\hline $\begin{array}{l}\text { Rosa } \\
2006^{77}\end{array}$ & Brazil & 24 & $\begin{array}{l}\text { - Inclusion }=\text { Consecutive patients with } \\
\text { COPD from pulmonary rehabilitation } \\
\text { center with: } \mathrm{PaO}_{2}=55 \mathrm{mmHg} \text { or } \mathrm{SpO}_{2} \\
=92 \% \text { (at rest and on room air), at least } 6 \\
\text { weeks of clinical stability, and satisfactory } \\
\text { ability to walk unaided. } \\
\text { - Exclusion = Patients with } \mathrm{SpO}_{2} \text { of } 80 \% \\
\text { during exercise, suffering from other } \\
\text { pulmonary diseases, heart diseases, } \\
\text { cardiac insufficiency or other comorbidities } \\
\text { considered uncontrolled or significant, and } \\
\text { those presenting formal contraindications } \\
\text { for performing exercise tests }\end{array}$ & $\begin{array}{l}\text { - Age }=67.8 \pm 7.5 \\
\text { - Male }=17 \\
\text { Female }=7 \\
\text { - BMI }=24.2 \pm 4.2 \\
\end{array}$ & $\begin{array}{l}\mathrm{n} \text { in GOLD stage of } \\
\text { COPD: } \\
\text { - Stage I mild: } 2 \\
\text { - Stage II moderate: } \\
7 \\
\text { - Stage III severe: } 12 \\
\text { - Stage IV: } 3\end{array}$ & $\begin{array}{l}-\mathrm{FEV}_{1} \\
\% \text { predicted } \\
=48.6 \pm 21.0 \\
\text { - FVC, } \\
\% \text { predicted } \\
=80.9 \pm 21.0\end{array}$ \\
\hline $\begin{array}{l}\text { Cote } \\
2007^{47}\end{array}$ & $\begin{array}{l}\text { United } \\
\text { States }\end{array}$ & 365 & $\begin{array}{l}\text { - Inclusion }=\text { Consecutive patients with } \\
\text { COPD recruited to the BODE protocol } \\
\text { between } 1994 \text { and } 2005 \text {, with smoking } \\
\text { history }>10 \text { pack years, FEV } 1 / F V C<0.70 \text {, } \\
\text { response to bronchodilation of } 12 \% \text { or } 200 \\
\text { mL, clinically stable for preceding } 6 \text { weeks } \\
\text { - Exclusion = Not stated }\end{array}$ & $\begin{array}{l}\text { - Age }=67 \pm 8 \\
\text { - Male /Female } \\
=\text { "mostly men" } \\
\text { - BMI = } 26.8 \pm 5.4\end{array}$ & $\begin{array}{l}\text { "Wide range of } \\
\text { COPD severity" }\end{array}$ & $\begin{array}{l}-\mathrm{FEV}_{1} \\
\mathrm{~L}=1.2 \pm 0.48 \\
\text { - } \mathrm{FEV}_{1} \\
\% \text { predicted } \\
=40 \pm 14 \\
\text { - FVC, } \\
\mathrm{L}=2.8 \pm 0.79\end{array}$ \\
\hline $\begin{array}{l}\text { Emtner } \\
2007^{51}\end{array}$ & Sweden & 21 & $\begin{array}{l}\text { - Inclusion = Consecutive patients who } \\
\text { had been admitted to hospital with acute } \\
\text { exacerbation of COPD; patients split into } \\
2 \text { groups for baseline parameters, but not } \\
\text { for analyses } \\
\text { - Exclusion = Not stated }\end{array}$ & $\begin{array}{l}\text { No hospitalization } \\
\text { group }(\mathrm{n}=12) \text { : } \\
\text { - Age }=65 \pm 10 \\
\text { - Male }=2 \\
\text { Female }=10 \\
\text { - BMI }=23 \pm 4 \\
\text { Hospitalization } \\
\text { group }(\mathrm{n}=9 \text { ): } \\
\text { - Age }=65 \pm 9 \\
\text { - Male }=5 \\
\text { Female }=4 \\
\text { - BMI }=22 \pm 4\end{array}$ & Not stated & $\begin{array}{l}\cdot \mathrm{FEV}_{1} \\
\% \text { predicted } \\
=40 \pm 12 \\
32 \pm 17\end{array}$ \\
\hline
\end{tabular}




\begin{tabular}{|c|c|c|c|c|c|c|}
\hline $\begin{array}{l}\text { Brown } \\
2008^{41}\end{array}$ & $\begin{array}{l}\text { United } \\
\text { States }\end{array}$ & 218 & $\begin{array}{l}\text { - Inclusion }=\text { COPD patients enrolled in } \\
\text { National Emphysema Treatment Trial with } \\
\text { radiographic evidence of emphysema, } \mathrm{FEV}_{1} \\
\leq 45 \% \text { predicted, TLC } \geq 100 \% \text { predicted, } \\
\mathrm{RV} \geq 150 \% \text { predicted and } \mathrm{PaCO}_{2} \leq 60 \\
\mathrm{mmHg} \text {, who had not smoked in prior } 4 \\
\text { months and did not have severe co-morbid } \\
\text { conditions } \\
\text { - Exclusion = Not stated }\end{array}$ & $\begin{array}{l}\text { - } \text { Age }=66.6 \pm 6.13 \\
\text { - } \text { Male }=746 \\
\text { Female }=472 \\
\text { - } \text { BMI = } 24.7 \pm 3.88\end{array}$ & $\begin{array}{l}\text { COPD patients with } \\
\text { severe or very severe } \\
\text { emphysema who } \\
\text { were participating in } \\
\text { a trial of lung } \\
\text { volume reduction } \\
\text { surgery }\end{array}$ & $\begin{array}{l}\text { - } \mathrm{FEV}_{1} \\
\mathrm{~L}=0.77 \pm 0.24 \\
\text { - } \mathrm{FEV}_{1} \\
\% \text { predicted } \\
=26.9 \pm 7.12 \\
\text { - FVC, } \\
\mathrm{L}=2.50 \pm 0.78 \\
\text { - FVC, } \\
\% \text { predicted } \\
=66.8 \pm 15.2\end{array}$ \\
\hline $\begin{array}{l}\text { Hill } \\
2008^{55}\end{array}$ & $\begin{array}{l}\text { Australia/ } \\
\text { Canada }\end{array}$ & 50 & $\begin{array}{l}\text { - Inclusion = Patients with diagnosis of } \\
\text { COPD, smoking history }>10 \text { pack years, } \\
\text { and } 15 \%<\mathrm{FEV}_{1} \text { predicted }<70 \% \\
\text { - Exclusion = History of lung surgery or } \\
\text { spontaneous pneumothorax, use of gait } \\
\text { aids or long-term oxygen therapy, any } \\
\text { comorbid condition thought to adversely } \\
\text { affect exercise performance (e.g., } \\
\text { musculoskeletal conditions, symptomatic } \\
\text { ischemic heart disease, neurologic or } \\
\text { cognitive impairment), BMI }>35 \mathrm{~kg} / \mathrm{m}^{2} \text {, or } \\
\text { tapering doses of corticosteroids or } \\
\text { methylxanthines }\end{array}$ & $\begin{array}{l}\text { - Age }=68 \pm 8 \\
\text { - Male = 36, } \\
\text { Female = } 14 \\
\text { - } \text { BMI = 23.4 } \pm 3.5\end{array}$ & Not stated & $\begin{array}{l}\text { - } \mathrm{FEV}_{1} \\
\mathrm{~L}=1.0 \pm 0.4 \\
\text { - } \mathrm{FEV}_{1} \\
\% \text { predicted } \\
=37 \pm 11 \\
\text { - } \mathrm{FEV}_{1} / \mathrm{FVC} \\
\%=37 \pm 9\end{array}$ \\
\hline $\begin{array}{l}\text { Pitta } \\
2008^{79}\end{array}$ & Brazil & 40 & $\begin{array}{l}\text { - Inclusion = Consecutive patients in } \\
\text { screening process for PR program between } \\
\text { June } 2006 \text { and June } 2007 \text { with clinically } \\
\text { stable COPD, and no osteo-neuro- } \\
\text { muscular comorbidities that might } \\
\text { interfere with assessments } \\
\text { - Exclusion = Patients not able to finalize } \\
\text { proposed assessments }\end{array}$ & $\begin{array}{l}\text { - Age }=68 \pm 7 \\
\text { - Male = } 21 \\
\text { Female }=19 \\
\cdot \text { BMI = } 24 \pm 6\end{array}$ & $\begin{array}{l}\mathrm{n} \text { in each GOLD } \\
\text { stage of COPD: } \\
\text { - Stage I mild: } 1 \\
\text { - Stage II moderate: } \\
11 \\
\text { - Stage III severe: } 19 \\
\text { - Stage IV: } 9\end{array}$ & $\begin{array}{l}\text { - } \mathrm{FEV}_{1} \\
\mathrm{~L}=0.90 \pm 0.26 \\
\text { - } \mathrm{FEV}_{1} \\
\% \text { predicted } \\
=41 \pm 14 \\
\text { - FVC, } \\
\% \text { predicted } \\
=66 \pm 19\end{array}$ \\
\hline $\begin{array}{l}\text { Brasil } \\
\text { Santos } \\
2009^{40}\end{array}$ & Brazil & 91 & $\begin{array}{l}\text { - Inclusion = Patients with COPD as } \\
\text { outpatients from pulmonary unit during } \\
2004 \text { whose records were chosen randomly } \\
\text { and then filtered according to following } \\
\text { exclusion criteria } \\
\text { - Exclusion = Patients enrolled in or who } \\
\text { had taken part in a pulmonary } \\
\text { rehabilitation program in preceding } 6 \\
\text { months, or those with skeletal-muscular } \\
\text { dysfunctions that prevented normal } \\
\text { walking, orthopedic appliances and/or } \\
\text { prostheses, hemodynamic instability with } \\
\text { infection or acute episodes in preceding } 8 \\
\text { weeks, or with prolonged oxygen therapy }\end{array}$ & $\begin{array}{l}\text { - Age }=64.4 \pm 8.5 \\
\text { - Male/Female } \\
=\text { Not stated } \\
\text { - BMI = Not stated }\end{array}$ & $\begin{array}{l}\text { COPD of all levels } \\
\text { of obstruction as per } \\
\text { GOLD guidelines }\end{array}$ & $\begin{array}{l}\text { - } \mathrm{FEV}_{1} \\
\% \text { predicted } \\
=63.4 \pm 25.3 \\
\text { - } \mathrm{FEV}_{1} / \mathrm{FVC} \\
\%=50.8 \pm 12.4\end{array}$ \\
\hline $\begin{array}{l}\text { Camargo } \\
2010^{42}\end{array}$ & Brazil & 50 & 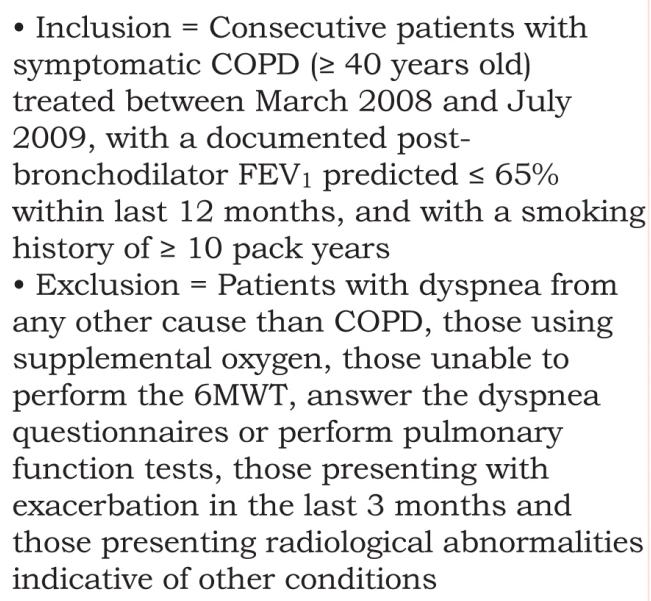 & $\begin{array}{l}\text { - Age }=69 \pm 8 \\
\text { - Male }=35 \\
\text { Female }=15 \\
\text { - BMI }=27 \pm 5 \\
\end{array}$ & Not stated & $\begin{array}{l}-\mathrm{FEV}_{1} \\
\mathrm{~L}=1.3 \pm 0.4 \\
\text { - } \mathrm{FEV}_{1} \\
\% \text { predicted } \\
=52 \pm 12 \\
\text { - FVC, } \\
\mathrm{L}=2.7 \pm 0.7 \\
\text { - FVC, } \\
\% \text { predicted } \\
=85 \pm 14\end{array}$ \\
\hline
\end{tabular}




\begin{tabular}{|c|c|c|c|c|c|c|}
\hline $\begin{array}{l}\text { Kozu } \\
2010^{57}\end{array}$ & Japan & 45 & $\begin{array}{l}\text { - Inclusion = Patients with COPD who were } \\
\text { clinically stable and had had no changes in } \\
\text { medication for at least } 4 \text { weeks prior to the } \\
\text { study } \\
\text { - Exclusion = Patients with very mild } \\
\text { symptoms, or those who had comorbid } \\
\text { conditions affecting exercise performance } \\
\text { (e.g. muscloskeletal or neurological } \\
\text { impairment, cardiac disease, severe } \\
\text { cognitive impairment) }\end{array}$ & $\begin{array}{l}\text { Age }=67.3 \pm 5.1 \\
\text { Male }=38 \\
\text { Female = } 7 \\
\text { BMI = } 20.8 \pm 2.2\end{array}$ & $\begin{array}{l}\text { Diagnosis of COPD } \\
\text { was made according } \\
\text { to established } \\
\text { criteria (GOLD) }\end{array}$ & $\begin{array}{l}\text { - } \mathrm{FEV}_{1} \\
\mathrm{~L}=1.10 \pm 0.50 \\
\text { - } \mathrm{FEV}_{1} \\
\% \text { predicted } \\
=45 \pm 12 \\
\text { - FVC, } \\
\% \text { predicted } \\
=81 \pm 22\end{array}$ \\
\hline $\begin{array}{l}\text { Fujimoto } \\
2011^{52}\end{array}$ & Japan & 130 & $\begin{array}{l}\text { - Inclusion = Consecutive middle-aged and } \\
\text { elderly outpatients with COPD recruited } \\
\text { between April } 1997 \text { and October } 2009 \\
\text { - Exclusion = Patients who had had } \\
\text { exacerbation or uncontrolled comorbidities, } \\
\text { such as malignancy, cardiovascular } \\
\text { disease, cerebrovascular disease, and } \\
\text { active musculoskeletal disease, during } 6 \\
\text { weeks preceding measurements }\end{array}$ & $\begin{array}{l}\text { - Age }=72.14 \pm 7.37 \\
\text { - Male = } 121 \\
\text { Female }=9 \\
\text { - } \mathrm{BMI}=21.05 \pm 3.68 \\
\end{array}$ & $\begin{array}{l}\text { n in GOLD stage of } \\
\text { COPD: } \\
\text { - Stage I mild: } 44 \\
\text { - Stage II moderate: } \\
53 \\
\text { - Stage III severe: } 29 \\
\text { - Stage IV: } 4\end{array}$ & $\begin{array}{l}-\mathrm{FEV}_{1} \\
\mathrm{~L}=1.28 \pm 0.52 \\
\text { - } \mathrm{FEV}_{1} \\
\% \text { predicted } \\
=70.61 \pm 28.03\end{array}$ \\
\hline $\begin{array}{l}\text { Annegarn } \\
2012^{36}\end{array}$ & $\begin{array}{l}\text { Nether- } \\
\text { lands }\end{array}$ & 79 & $\begin{array}{l}\text { - Inclusion = Patients with COPD recruited } \\
\text { from pre-rehabilitation assessment } \\
\text { - Exclusion = Patients who had had } \\
\text { exacerbation-related hospitalization within } \\
4 \text { weeks prior to assessment, who used a } \\
\text { rollator, or who were unable to complete at } \\
\text { least one } 6 \mathrm{MWT} \text { without stopping }\end{array}$ & $\begin{array}{l}- \text { Age }=64.3 \pm 8.9 \\
\text { - } \text { Male }=47 \\
\text { Female }=32 \\
\cdot \text { BMI = } 24.7 \pm 4.5\end{array}$ & $\begin{array}{l}\mathrm{n} \text { in GOLD stage of } \\
\text { COPD: } \\
\text { - Stage I mild: } 8 \\
\text { - Stage II moderate: } \\
36 \\
\text { - Stage III severe: } 28 \\
\text { - Stage IV very } \\
\text { severe: } 7\end{array}$ & $\begin{array}{l}\text { - } \mathrm{FEV}_{1} \text {, } \\
\% \text { predicted } \\
=53.5 \pm 18.7\end{array}$ \\
\hline $\begin{array}{l}\text { Chen L } \\
2012^{74}\end{array}$ & Taiwan & 17 & $\begin{array}{l}\text { - Inclusion = Patients with clinically stable } \\
\text { COPD without baseline oxygen saturation } \\
\text { recruited from July } 2008 \text { to July } 2010 \\
\text { who were } 60-80 \text { years old, non-smokers } \\
\text { or smoking history of } 10 \text { pack years, with } \\
\text { no pathology that could interfere with the } \\
\text { ability to perform exercises } \\
\text { - Exclusion = Not stated }\end{array}$ & $\begin{array}{l}\text { - Age = } 73.5 \pm 5.7 \\
\text { - Male = 17, } \\
\text { Female = 0 } \\
\text { - } \text { BMI = 22.3 } \pm 3.5\end{array}$ & $\begin{array}{l}\mathrm{n} \text { in GOLD stage of } \\
\text { COPD: } \\
\text { - Stage I mild: } 1 \\
\text { - Stage II moderate: } \\
6 \\
\text { - Stage III severe: } 6 \\
\text { - Stage IV very } \\
\text { severe: } 4\end{array}$ & $\begin{array}{l}\text { - } \mathrm{FEV}_{1} \\
\mathrm{~L}=0.9 \pm 0.1 \\
\text { - } \mathrm{FEV}_{1} \\
\% \text { predicted } \\
=43.1 \pm 16.2 \\
\text { - FVC, } \\
\mathrm{L}=1.7 \pm 0.1 \\
\text { - FVC, } \\
\% \text { predicted } \\
=54.5 \pm 13.2\end{array}$ \\
\hline $\begin{array}{l}\text { Chen S } \\
2012^{44}\end{array}$ & Taiwan & 37 & $\begin{array}{l}\text { - Inclusion = COPD patients with chronic } \\
\text { hypercapnia, } \mathrm{FEV}_{1}<50 \% \text { predicted, } \\
\text { daytime awake } \mathrm{PaCO}_{2}>45 \mathrm{mmHg}, \mathrm{PaO}_{2} \\
<80 \mathrm{mmHg} \text {, } \mathrm{mH} 7.30-7.45 \text { with room air, } \\
\text { medical stability in preceding } 3 \text { months, } \\
\text { good motivation to participate in study, } \\
\text { and have not participated in pulmonary } \\
\text { rehabilitation programs } \\
\text { - Exclusion = Patients who could not } \\
\text { perform } 6 \mathrm{MWT} \text { owing to various other } \\
\text { diseases (e.g., orthopedic or } \\
\text { neuromuscular problems or other } \\
\text { systematic diseases) and were } \\
\text { uncooperative or poorly motivated to } \\
\text { participate }\end{array}$ & $\begin{array}{l}\text { - Age }=64.4 \pm 10.9 \\
\text { - Male = } 28 \\
\text { Female }=9 \\
\text { - } \mathrm{BMI}=21.4 \pm 4.8\end{array}$ & Not stated & $\begin{array}{l}\text { - FVC, } \\
\mathrm{L}=1.2 \pm 0.5 \\
\text { - FVC, } \\
\% \text { predicted } \\
=41.4 \pm 17.1 \\
\text { - } \mathrm{FEV}_{1}, \\
\mathrm{~L}=0.6 \pm 0.20 \\
\text { - } \mathrm{FEV}_{1}, \\
\% \text { predicted } \\
=26.1 \pm 7.7 \\
\text { - } \mathrm{FEV}_{1} / \mathrm{FVC}, \\
\%=50.9 \pm 12.7\end{array}$ \\
\hline $\begin{array}{l}\text { Waatevik } \\
2012^{75}\end{array}$ & Norway & 370 & $\begin{array}{l}\text { - Inclusion = Patients with COPD included } \\
\text { in the previously published Bergen COPD } \\
\text { cohort study who were able to complete a } \\
6 \mathrm{MWT} \text { without stopping and without } \\
\text { supplemental oxygen } \\
\text { - Exclusion = Not stated }\end{array}$ & $\begin{array}{l}\text { - Age }=63.3 \pm 6.8 \\
\text { - Male = 223, } \\
\text { Female = } 147 \\
\text { - BMI = Not stated }\end{array}$ & Not stated & $\begin{array}{l}\text { - } \mathrm{FEV}_{1} \text {, } \\
\% \text { predicted } \\
=50.4 \pm 13.8\end{array}$ \\
\hline
\end{tabular}


ATS=American Thoracic Society; BMI=body mass index; BODE=Body mass index, airflow Obstruction, Dyspnea and Exercise capacity index; BTS=British Thoracic Society; $\mathrm{COPD}=$ chronic obstructive pulmonary disease; ERS=European Respiratory Society; $\mathrm{FEV}_{1}=$ forced expiratory volume in 1 second; FVC=forced vital capacity; GOLD, Global initiative for chronic Obstructive Lung Disease; HR=heart rate; IQR=interquartile range; ISWT=incremental shuttle walk test; IVC=inspiratory slow vital capacity; $\mathrm{kPa}=\mathrm{kilopascal;} \mathrm{L=litres;}$

6MWT=6-minute walk test; $\mathrm{MRC}=$ Medical Research Council; $\mathrm{MVV}=$ maximal voluntary ventilation; $\mathrm{NR}_{\mathrm{n}}$ not reported; $\mathrm{PaCO}=$ partial pressure of arterial carbon dioxide; $\mathrm{PaO}_{2}=$ partial pressure of arterial oxygen; $\mathrm{PR}=$ pulmonary rehabilitation; RER=respiratory exchange ratio; $\mathrm{RV}=$ residual volume; $\mathrm{SD}=$ standard deviation; $\mathrm{SpO}_{2}=$ arterial oxygen saturation; $\mathrm{TLC}=$ total lung capacity; $\mathrm{VC}=$ vital capacity.

Values presented are mean \pm standard deviation unless otherwise stated.

significant Pearson's correlations (very weak to moderate; $r=-0.16-0.41$ ), with 1 study $^{38}$ reporting a negative correlation. The remaining 2 studies reported that Pearson's correlations were not significant. ${ }^{61,64}$

FVC and ICET, TT and $\mathrm{VO}_{2}$

Of 4 studies $45,49,59,62$ assessing associations between FVC and peak $\mathrm{VO}_{2}$ obtained during ICET, 3 studies ${ }^{45,49,59}$ presented significant Pearson's correlations (strong; $r=0.54-0.67$ ); the remaining study reported that Pearson's correlations were not significant. $^{62}$ One further study reported a significant Pearson's correlation between FVC and Wmax achieved during ICET (moderate; $r=0.58) .{ }^{41}$ One other study reported a significant Pearson's correlation between FVC and peak $\mathrm{VO}_{2}$ during TT (strong; $r=0.63$ ). ${ }^{70}$

\section{Correlations Between Exercise Test Outcomes and IC}

Baseline IC was reported in 6 studies (Table 3; Figure 2c). $41,42,50,52,73,74$

\section{IC and 6MWT}

Of these, all 3 studies ${ }^{41,52,74}$ assessing Pearson's correlations for IC and the 6MWT found significant relationships (weak to moderate; $r=0.38-0.62$ ); a further 2 studies also reported significant Spearman's values for this relationship (moderate; $\rho=0.51$ and 0.57 ). ${ }^{42,73}$

\section{IC and ISWT, ICET and TT}

Significant moderate correlations were also reported between IC and ISWT $(\rho=0.50)$, and $W_{\max }$ during ICET $(r=0.59)$ and $\mathrm{TT}(\rho=0.48) .{ }^{50}$

\section{Correlations Between Exercise Test Outcomes and Exercise-induced Changes in $\mathrm{PaO}_{2}$}

A total of 14 studies assessed correlations between change in $\mathrm{PaO}_{2}$ and exercise test outcomes (Table 3; Figure 2d).
$\mathrm{PaO}_{2}$ and $6 \mathrm{MWT}$

Three $44,47,75$ of 5 studies $39,40,44,47,75$ studies assessing Pearson's correlations between 6MWT and $\mathrm{PaO}_{2}$ reported significant correlations (very weak to weak; $r=0.15-0.35$ ), with the 2 remaining studies 39,40 reporting no significant correlation. Of 3 studies $63,76,77$ reporting Spearman's correlations between 6MWT and $\mathrm{PaO}_{2}, 2$ studies ${ }^{63,76}$ found no significant association and 1 study ${ }^{77}$ found a significant correlation (moderate; $\rho=0.42)$.

\section{$\mathrm{PaO}_{2}$ and $12 \mathrm{MWT}$}

Pearson's correlations for $12 \mathrm{MWT}$ were assessed in 4 studies $^{48,58,68,78}$ : 2 studies ${ }^{48,68}$ reported moderate correlations $(r=0.42-0.44)$ with the remaining 2 studies $^{58,78}$ reporting no correlation.

\section{$\mathrm{PaO}_{2}$ and ISWT}

Of 3 studies reporting Spearman's correlations for the ISWT, ${ }^{50,76,77} 2$ studies ${ }^{50,77}$ found moderate correlations ( $\rho=0.42-0.53$ ) with 1 study $^{76}$ reporting no correlation. In the 3 studies assessing ICET (peak $\left.\mathrm{VO}_{2}\right),{ }^{47,63,80} 2$ studies ${ }^{47,80}$ reported only weak Pearson's correlations $(r=0.21-0.28)$ with 1 study ${ }^{63}$ reporting no Spearman's correlation.

\section{Correlations Between Exercise Test Outcomes and TLC}

Additionally, it was anticipated that associations between exercise and TLC would be included in the review. However, too few studies were found and therefore not included in the final results.

\section{Discussion}

This study has shown that there are limited data supporting strong correlations between exercise test outcomes and commonly used assessments of lung function. FEV 1 appears to correlate well with the outcomes of the ICET (both $\mathrm{VO}_{2}$ and $\mathrm{W}_{\max }$ ). The association between the most commonly used field- 


\section{Table 3. Correlations Between Exercise Test Outcomes and Selected Physiological Variables}

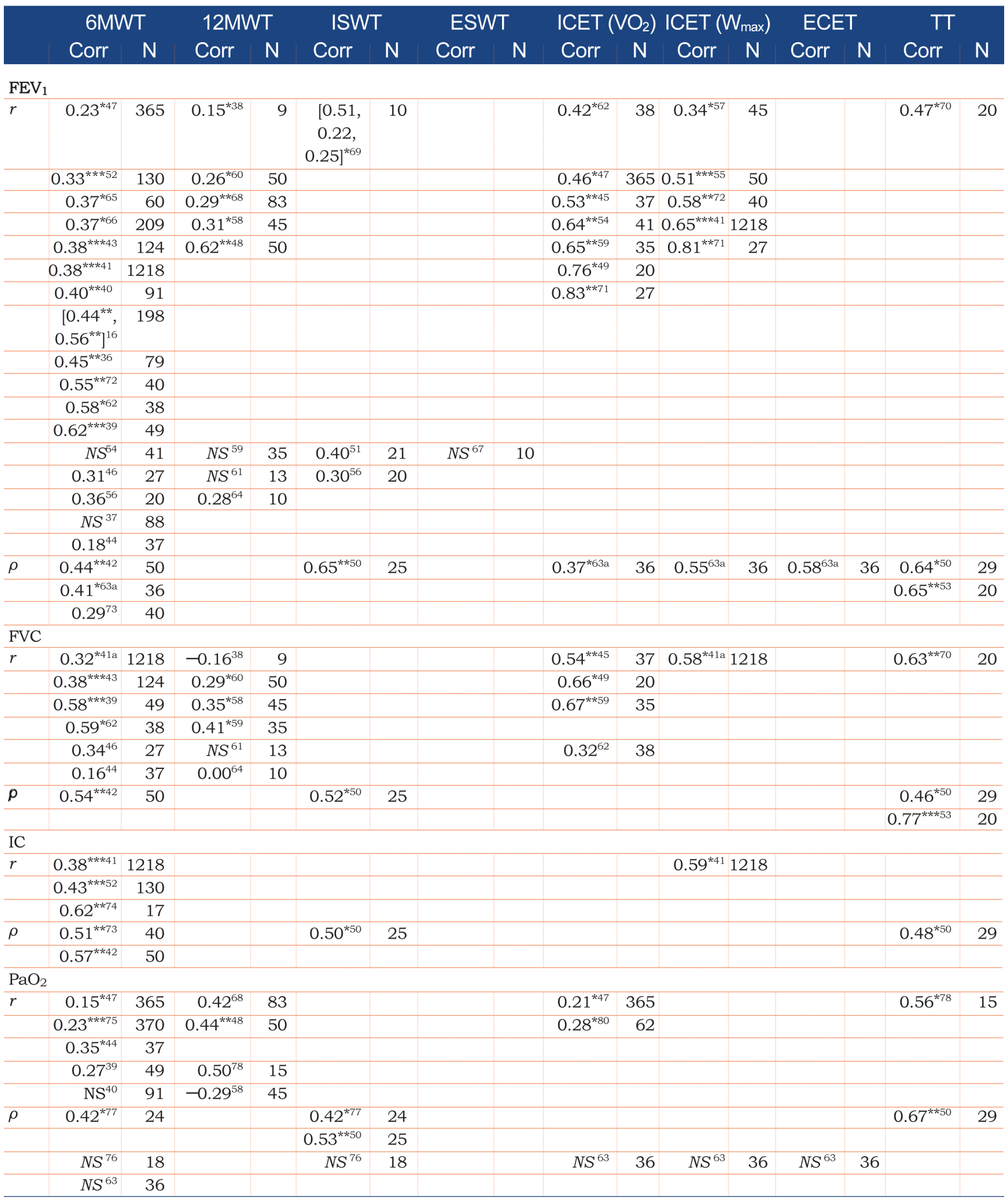




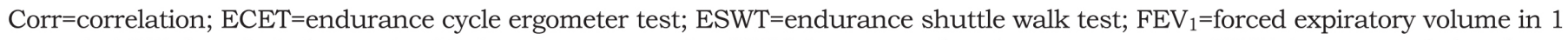
second; FVC=forced vital capacity; IC=inspiratory capacity; ICET=incremental cycle ergometer test; ISWT=incremental shuttle walk test; 6MWT=6-minute walk test; 12MWT=12-minute walk test; NS=studies reporting no significant correlation; $\rho=$ Spearman's rank coefficient; $\mathrm{PaO}_{2}=$ partial pressure of arterial oxygen; $r=$ Pearson's regression coefficient; TT=treadmill test; $\mathrm{VO}_{2}=$ oxygen consumption; $\mathrm{W}_{\max }=$ highest workload achieved.

Square parentheses enclose the results of different subgroups within the same study.

${ }^{*} p<0.05,{ }^{* *} p<0.01,{ }^{* *} p<0.001$, No $\left({ }^{*}\right)$, not significant $r$ value, NS not significant ( $r$ value not provided in original article) a significance assumed as $p<0.05$ from table inclusion criteria, actual value not given in text. However, only those $p$ values deviating from table inclusion criteria are shown.

based tests of exercise capacity, the 6MWT and 12MWT, and $F E V_{1}$ is unclear.

$\mathrm{FEV}_{1}$ is used as the main diagnostic criterion for COPD, ${ }^{4,22,23}$ and the European Medicines Agency also suggests that pre- and post-bronchodilator FEV $\mathrm{F}_{1}$, both at baseline and repeatedly during follow-up, is used to demonstrate the efficacy of therapeutic interventions in clinical trials. ${ }^{24} \mathrm{FEV}_{1}$ correlates better with laboratorybased tests such as the ICET (primarily moderate to very strong correlations) than with field-based tests, such as 6MWT and the 12MWT (although these do consistently demonstrate weak/moderate correlations). The ICET also appeared to have the closest relationship to FVC and IC, albeit with very limited evidence. Conversely, ICET correlated only weakly with $\mathrm{PaO}_{2}$ in studies reporting this relationship.

Some caution is warranted in placing too much emphasis on lung function alone as a gold standard assessment in COPD relative to exercise tests. While individual lung function measurements, such as FEV are used in diagnosing the severity of COPD and predicting mortality, ${ }^{27}$ it should be remembered that COPD patients have systemic disease manifestations that are not necessarily reflected by a single lung function result. Patients with similar FEV 1 may nevertheless have significantly different function defects not captured by this test. Exercise tests, in measuring whole lung functionality, may be expected to correlate imprecisely with individual lung function parameters. Furthermore, their design may capture the systemic aspects of COPD lung dysfunction more effectively and thus provide additional prognostic information. In fact, several prospective studies have shown that $6 \mathrm{MWT}$ is a better predictor of mortality than $\mathrm{FEV}_{1}$ in patients with severe $\operatorname{COPD}^{16,81}$ and coupling $6 \mathrm{MWT}$ output to individual lung function parameters like FEV 1 and $\mathrm{PaO}_{2}$ has proven utility ${ }^{82,83}$ and underpins the rationale for the multidimensional grading system for COPD, the BMI, airflow, Obstruction, Dyspnea and
Excercise capacity (BODE) index. ${ }^{84}$

The observation that the $6 \mathrm{MWT}$ and the $12 \mathrm{MWT}$ are the most often reported in conjunction with measures of lung function is unsurprising as they are well established, require little equipment, training or preparation, and (for the 6MWT at least) minimal, clinically important, difference reference values are available. Of the laboratory-based tests, the ICET is by far the most widely used. However, this serves to highlight the paucity of data reporting the relationship between other exercise tests and measures of lung function. When reported, for example, the ISWT and TT exhibited mostly moderate to good correlations with the 4 physiological parameters assessed in this review. However, it is difficult to draw definitive conclusions about the applicability of these tests when the data are so rarely reported. Correlations between the FEV $1, F V C$, IC and $\mathrm{PaO}_{2}$ and the ESWT and ECET have so seldom been reported that no meaningful interpretation of these relationships can be made.

Exercise tests, such as those reviewed here, are used to assess the exercise capacity of patients with COPD. These tests are important because the systemic consequences of COPD include reduced exercise capacity and ensuing decreases in physical activity. However, the findings of this systematic review suggest that the relationships between exercise and $F E V_{1}$, FVC, IC and $\mathrm{PaO}_{2}$ are under-reported for most tests, and even for the most commonly reported tests, these associations are often equivocal. This suggests that although the information obtained from these tests may be of use in assessing exercise tolerance, caution should be used before applying the results of these tests to make assessments of physiological effects of COPD. Exercise capacity appears to be such a multifactorial outcome that it is difficult to conclusively link test performance to any of the physiological variables reviewed. This supports a recent systematic review that qualitatively compared patients' performance in these 


\section{Figure 2. Ranges of Reported Pearson's Correlations}

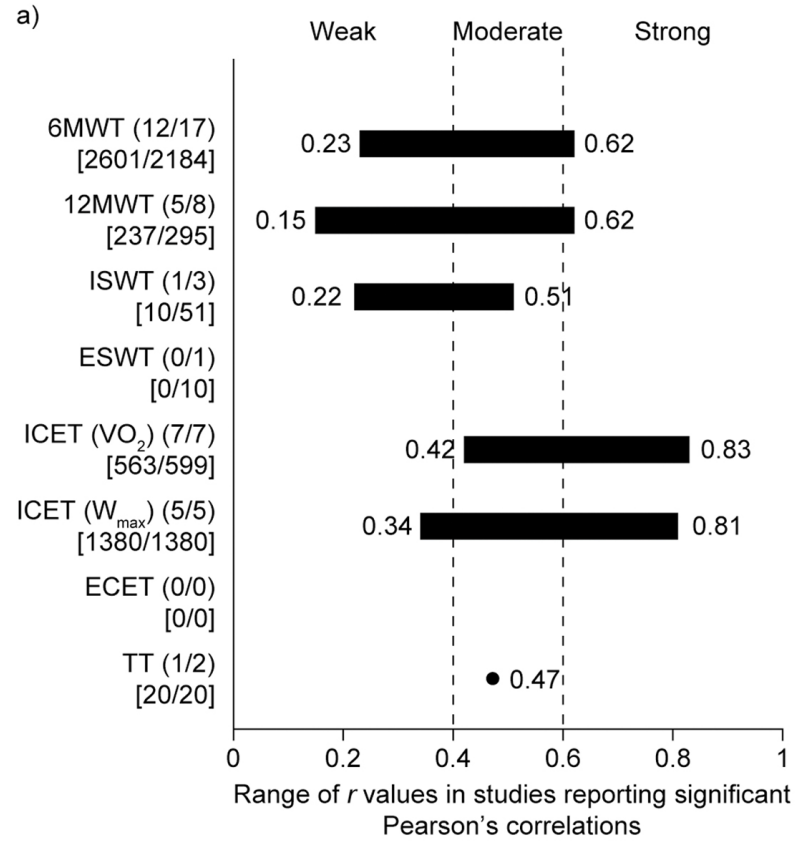

c)

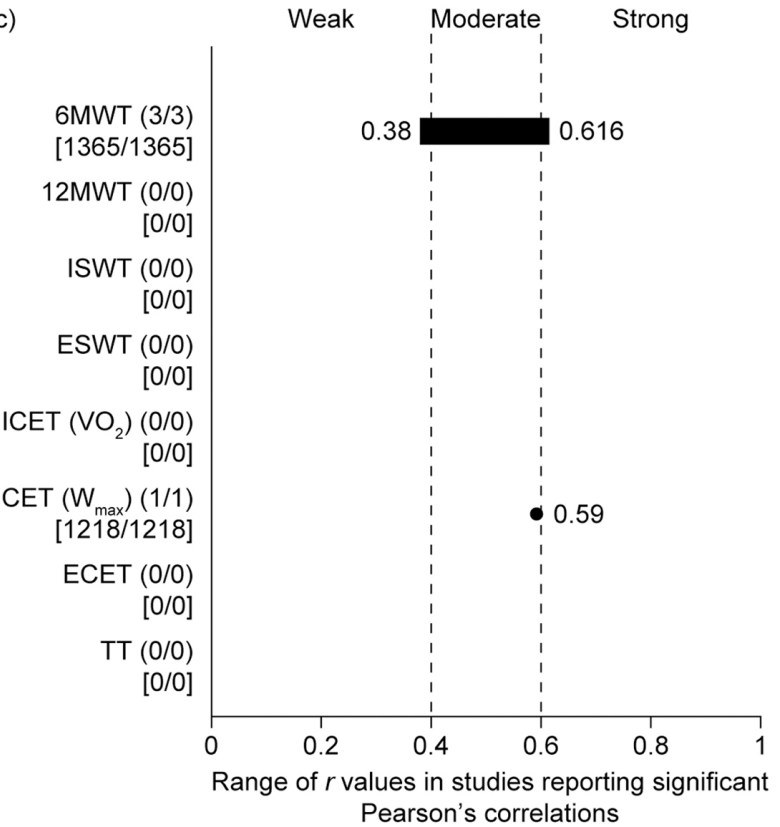

b) Strong Moderate Weak Moderate Strong
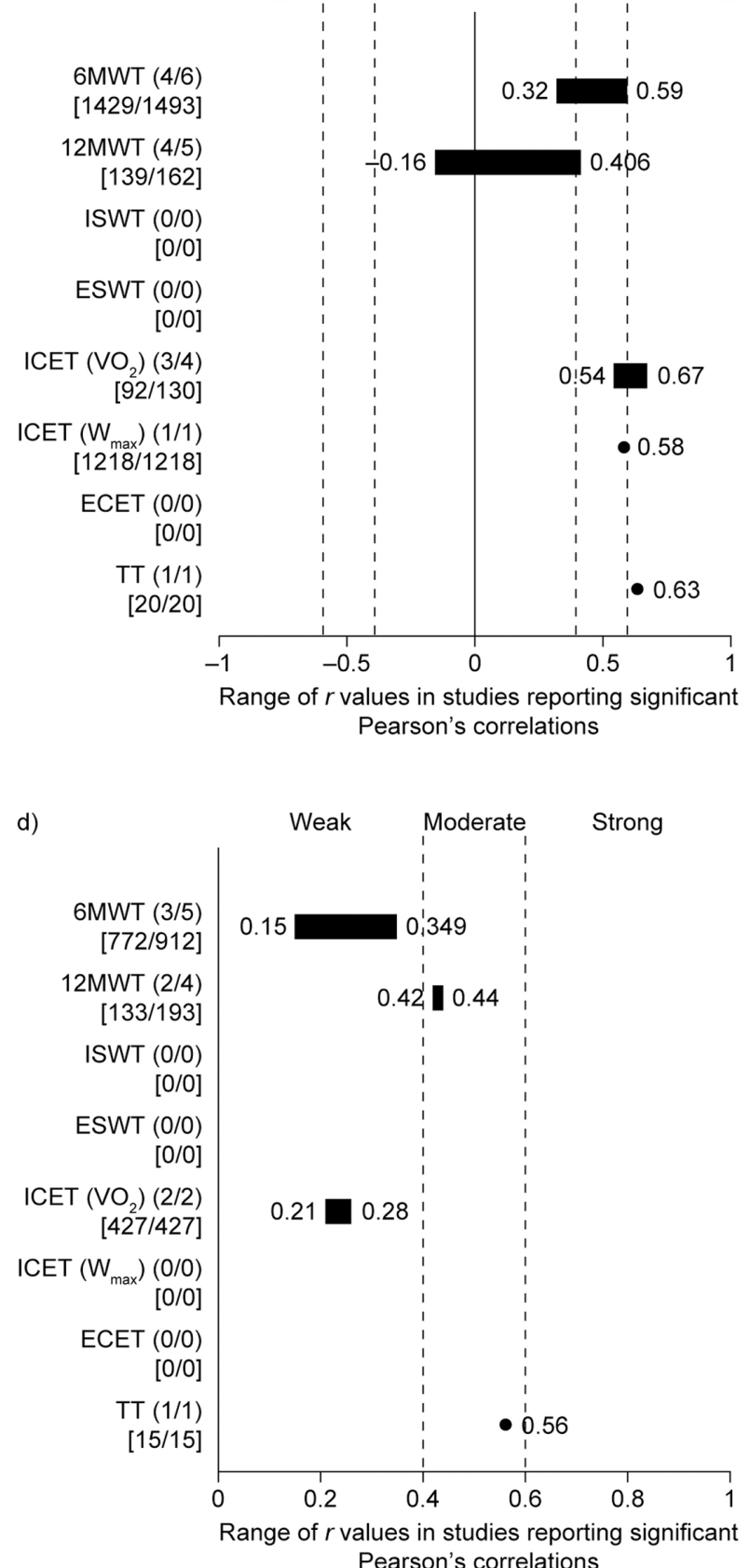

Ranges of reported Pearson's correlations in studies reporting significant associations between exercise tests and: a) FEV 1 ; b) FVC; c) IC and d) $\mathrm{PaO}_{2}$. Brackets indicate the number of studies reporting significant correlations/total number of studies reporting Pearson's

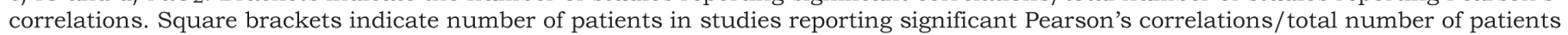
in studies assessing Pearson's correlations.

ECET=endurance cycle ergometer test; ESWT=endurance shuttle walk test; ICET=incremental cycle ergometer test; ISWT=incremental shuttle walk test; $6 \mathrm{MWT}=6$-minute walk test; $12 \mathrm{MWT}=12$-minute walk test; $r=$ Pearson's regression coefficient; TT=treadmill test; $\mathrm{VO}_{2}=$ oxygen consumption; $\mathrm{W}_{\max }=$ highest workload achieved. 
exercise tests and found no discernible advantage of any particular test. ${ }^{34}$

Limitations of this review include the wide range of study designs and patient cohorts involved. Furthermore, the association between lung function and exercise performance are most probably not adjusted for important confounders such as age, gender, height, comorbidities and weight. It is also possible that study results are confounded by limited patient numbers. Using the most commonly reported association as an example, the 12 studies reporting a significant Pearson's correlation between $6 \mathrm{MWT}$ and $\mathrm{FEV}_{1}$ had a median $n$ of 108 (range: 38-1218); the 5 studies that reported no significant association between these parameters had a median $n$ of 39 (range: 20-88). It is therefore possible that significant associations could be underreported owing to a type II statistical reporting error. On the other hand, it can be seen where $r / \rho$ are reported for correlations between the same 2 parameters, that there is a tendency for larger populations to have a lower value and associated lower significance, suggesting that smaller populations can over-emphasize a genuine relationship. Both of these factors must be considered when designing studies assessing these exercise tests as well as the ability of pharmacological interventions to affect their output. Other confounding variables that are difficult to control for in such a review include whether or not the guidelines from the ATS were strictly adhered to in all the tests. This is particularly important regarding the technical aspects of tests such as $6 \mathrm{MWT}$ where even small deviations in methodology can influence output. ${ }^{85}$ Finally, the inclusion criteria and COPD severity are often not clearly stated by the studies included in this review. Therefore, there is a risk that the patients in the included studies are not broadly homogenous. However, in this case the weakness lies in the reporting of studies, and we recommend that future studies clearly state inclusion criteria and clinical rationale for diagnosis whenever possible.

Recent guidelines on the diagnosis and treatment of COPD indicate that assessment of disease severity is improved by using functional criteria such as exercise capacity. $4,22,23$ However, no distinction is made in these guidelines between the different exercise tests.
For example, ICET and 6MWT output are thought to measure different physio-biochemical variables, ${ }^{86}$ and it has been argued that the latter is a better reflection of a patient's ability to carry out daily activities. ${ }^{87}$ The current findings suggest that clinicians or investigators wishing to assess exercise capacity in patients with COPD must carefully consider the physiological consequences of COPD when interpreting the results of these tests. In particular, based on our review of the available data, it is important not to choose an exercise test based solely on patient lung function. Rather, tests should be chosen based on the ability of a pharmaceutical agent to influence the test based on the effect that agent is anticipated to have. For example, an agent that primarily affects lungs and subsequently improves tests measuring lung function, volume and breathlessness may well differ from an agent anticipated to have more systemic consequences.

\section{Acknowledgements:}

All authors have contributed to the conception and design of the study, analysis and interpretation of data and revision of the manuscript. All authors approve the final version of the manuscript. Martin Bell and Iain Fotheringham of Oxford PharmaGenesis, Ltd., provided writing support funded by GlaxoSmithKline, Uxbridge, United Kingdom.

\section{Declaration of Interest:}

Yogesh Suresh Punekar, John Riley and Sarah Cockle are current employees of GlaxoSmithKline, Uxbridge, United Kingdom. Sally Singh was involved with the development of the incremental shuttle walk test, and has served on advisory boards for GlaxoSmithKline. Sally Singh was partially funded by the National Institute for Health Research (NIHR) Collaboration for Leadership in Applied Health Research and Care East Midlands. Support was also provided by the NIHR Leicester Respiratory Biomedical Research Unit. The views expressed are those of the authors and not necessarily those of the National Health Service (NHS), the NIHR or the Department of Health. 


\section{References}

1. Lopez AD, Shibuya K, Rao C, et al. Chronic obstructive pulmonary disease: current burden and future projections. Eur Respir J. 2006;27(2):397-412.

doi: http://dx.doi.org/10.1183/09031936.06.00025805

2. Halbert RJ, Natoli JL, Gano A, Badamgarav E, Buist AS, Mannino DM. Global burden of COPD: systematic review and meta-analysis. Eur Respir J. 2006;28(3):523-532. doi: http://dx.doi.org/10.1183/09031936.06.00124605

3. Mathers CD, Loncar D. Projections of global mortality and burden of disease from 2002 to 2030. PLoS Med. 2006;3(11):e442. doi: http://dx.doi.org/10.1371/journal.pmed.0030442

4. Global initiative for chronic Obstructive Lung Disease (GOLD). Global strategy for the diagnosis, management and prevention of COPD. GOLD website. http://www.goldcopd.org/. Published 2014. Accessed September 12, 2014.

5. Gosker HR, Lencer NHMK, Franssen FME, van der Vusse GJ, Wouters EF, Schols AM. Striking similarities in systemic factors contributing to decreased exercise capacity in patients with severe chronic heart failure or COPD. Chest. 2003;123(5):14161424. doi: http://dx.doi.org/10.1378/chest.123.5.1416

6. O'Donnell DE, Gebke KB. Activity restriction in mild COPD: a challenging clinical problem. Int J Chron Obstruct Pulmon Dis. 2014; 9: 577-588. doi: http://dx.doi.org/10.2147/COPD.S62766

7. Troosters T, Sciurba F, Battaglia S, et al. Physical inactivity in patients with COPD, a controlled multi-center pilot-study. Respir Med. 2010; 104(7): 1005-1011. doi: http://dx.doi.org/10.1016/j.rmed.2010.01.012

8. Diaz AA, Morales A, Diaz JC, et al. CT and physiologic determinants of dyspnea and exercise capacity during the sixminute walk test in mild COPD. Respir Med. 2013; 107(4): 570579. doi: http://dx.doi.org/10.1016/j.med.2012.12

9. Aliverti A, Macklem PT. How and why exercise is impaired in COPD. Respiration.2001;68(3): 229-239.

doi: http://dx.doi.org/10.1159/000050502

10. Wust RC, Degens H. Factors contributing to muscle wasting and dysfunction in COPD patients. Int J Chron Obstruct Pulmon Dis. 2007; 2(3): 289-300.

11. Jones P, Miravitlles M, van der Molen T, Kulich K. Beyond FEV(1) in COPD: a review of patient-reported outcomes and their measurement. Int J Chron Obstruct Pulmon Dis. 2012;7:697709. doi: http://dx.doi.org/10.2147/COPD.S32675

12. Jones PW. Issues concerning health-related quality of life in COPD. Chest. 1995;107(5 Suppl):187S-193S. doi: http://dx.doi.org/10.1378/chest.107.5_Supplement.187S

13. Cooper CB. The connection between chronic obstructive pulmonary disease symptoms and hyperinflation and its impact on exercise and function. Am J Med. 2006;119(10 Suppl 1):21-31. doi: http://dx.doi.org/10.1016/j.amjmed.2006.08.004

14. Mahler DA, Harver A. A factor analysis of dyspnea ratings, respiratory muscle strength, and lung function in patients with chronic obstructive pulmonary disease. Am Rev Respir Dis. 1992;145(2 Pt 1):467-470.

doi: http://dx.doi.org/10.1164/ajrccm/145.2_Pt_1.467
15. Ozgur ES, Nayci SA, Ozge C, Tasdelen B. An integrated index combined by dynamic hyperinflation and exercise capacity in the prediction of morbidity and mortality in COPD. Respiratory Care. 2012;57(9):1452-1459.

doi: http://dx.doi.org/10.4187/respcare.01440

16. Pinto-Plata VM, Cote C, Cabral H, Taylor J, Celli BR. The 6-min walk distance: change over time and value as a predictor of survival in severe COPD. Eur Respir J. 2004;23(1):28-33. doi: http://dx.doi.org/10.1183/09031936.03.00034603

17. Spruit MA, Polkey MI, Celli B, et al. Predicting outcomes from 6-Minute walk distance in chronic obstructive pulmonary disease. J Am Med Dir Assoc. 2012;13(3):291-297. doi: http://dx.doi.org/10.1016/j.jamda.2011.06.009

18. Oga T, Nishimura K, Tsukino M, Sato S, Hajiro T. Analysis of the factors related to mortality in chronic obstructive pulmonary disease: role of exercise capacity and health status. Am J Respir Crit Care Med. 2003;167(4):544-549. doi: http://dx.doi.org/10.1164/rccm.200206-5830C

19. Tojo N, Ichioka M, Chida M, Miyazato I, Yoshizawa Y, Miyasaka N. Pulmonary exercise testing predicts prognosis in patients with chronic obstructive pulmonary disease. Intern Med. 2005;44(1):20-25.

doi: http://dx.doi.org/10.2169/internalmedicine.44.20

20. Ringbaek T, Martinez G, Brøndum E, Thøgersen J, Morgan M, Lange P. Shuttle walking test as predictor of survival in chronic obstructive pulmonary disease patients enrolled in a rehabilitation program. J Cardiopulmon Rehabil Prev. 2010;30(6):409-414.

doi: http://dx.doi.org/10.1097/HCR.0b013e3181e1736b

21. Williams JEA, Green RH, Warrington V, Steiner MC, Morgan MD, Singh SJ. Development of the i-BODE: Validation of the incremental shuttle walking test within the BODE index. Respir Med. 2012;106(3):390-396. doi: http://dx.doi.org/10.1016/j.rmed.2011.09.005

22. American Thoracic Society/European Respiratory Society Task Force. Standards for the Diagnosis and Management of Patients with COPD, Version 1.2. American Thoracic Society website. http://www.thoracic.org/go/copd. Published 2005. Accessed May 19, 2014.

23. National Institute for Health and Clinical Excellence (NICE). Management of chronic obstructive pulmonary disease in adults in primary and secondary care (partial update). NICE website. http://guidance.nice.org.uk/cg101. Published 2010. Accessed May 19, 2014.

24. European Medicines Agency. Guidelines on clinical investigation of medicinal products in the treatment of chronic obstructive pulmonary disease (COPD). European Medicines Agency website. http://www.ema.europa.eu/docs/ en_GB/document_library/Scientific_guideline/2012/08/ WC500130880.pdf. Published 2012. Accessed July 2014.

25. Pichurko BM. Exercising your patient: which test(s) and when? Respir Care. 2012; 57(1): 100-110. doi: http://dx.doi.org/10.4187/respcare.01428

26. Miller MR, Hankinson J, Brusasco V, et al. Standardisation of spirometry. Eur Respir J. 2005; 26(2):319-338. doi: http://dx.doi.org/10.1183/09031936.05.00034805

27. Hansen EF, Phanareth K, Laursen LC, Kok-Jensen A, Dirksen A. Reversible and irreversible airflow obstruction as predictor of overall mortality in asthma and chronic obstructive pulmonary disease. Am J Respir Crit Care Med. 1999; 159 (4 Pt 1): 1267-1271. doi: http://dx.doi.org/10.1164/ajrccm.159.4.9807121 
28. Jones PW. Health status and the spiral of decline. COPD. 2009; 6(1): 59-63. doi: http://dx.doi.org/10.1080/15412550802587943

29. Trzaska-Sobczak M, Brozek G, Farnik M, Pierzchala W. Evaluation of COPD progression based on spirometry and exercise capacity. Pneumonol Alergol Pol. 2013;81(4):288-293.

30. Cerveri I, Pellegrino R, Dore R, et al. Mechanisms for isolated volume response to a bronchodilator in patients with COPD. $J$ Appl Physiol (1985). 2000; 88(6): 1989-1995.

31. Parker CM, Voduc N, Aaron SD, Webb KA, O’Donnell DE. Physiological changes during symptom recovery from moderate exacerbations of COPD. Eur Repir J. 2005; 26(3): 420-428. doi: http://dx.doi.org/10.1183/09031936.05.00136304

32. Casanova C, Cote C, de Torres JP, et al. Inspiratory-to-lung capacity ratio predicts mortality in patients with chronic obstructive pulmonary disease. Am J Respir Crit Care Med. 2005; 171(6): 591-597.

doi: http://dx.doi.org/10.1164/rccm.200407-8670C

33. Gay PC. Chronic obstructive pulmonary disease and sleep. Respir Care. 2004: 49 (1): 39-51.

34. Fotheringham I, Meakin G, Punekar YS, Riley JH, Cockle SM, Singh SJ. Comparison of laboratory-and field-based exercise tests for COPD: a systematic reivew. Int J Chron Obstruct Pulmon Dis. 2015; 10:625-643. doi: http://dx.doi.org/10.2147/COPD.S70518

35. Moher D, Liberati A, Tetzlaff J, Altman DG for the PRISMA Group. Preferred reporting items for systematic reviews and meta-analyses: the PRISMA statement. BMJ. 2009; 339: b2535. doi: http://dx.doi.org/10.1136/bmj.b2535

36. Annegarn J, Spruit MA, Savelberg HHCM, et al. Differences in walking pattern during 6-min walk test between patients with COPD and healthy subjects. PLoS ONE. 2012;7(5). doi: http://dx.doi.org/10.1371/journal.pone.0037329

37. Behnke M, Wewel AR, Kirsten D, Jorres RA, Magnussen H. Exercise training raises daily activity stronger than predicted from exercise capacity in patients with COPD. Respir Med. 2005;99(6):711-717.

doi: http://dx.doi.org/10.1016/j.rmed.2004.10.016

38. Bernstein ML, Despars JA, Singh NP, Avalos K, Stansbury DW, Light RW. Reanalysis of the 12-minute walk in patients with chronic obstructive pulmonary disease. Chest. 1994;105(1):163167. doi: http://dx.doi.org/10.1378/chest.105.1.163

39. Borak J, Chodosowska E, Matuszewski A, Zielinski J. Emotional status does not alter exercise tolerance in patients with chronic obstructive pulmonary disease. Eur Respir J. 1998;12(2):370-373.

40. Brasil Santos D, de Assis Viegas CA. Correlation of levels of obstruction in COPD with lactate and six-minute walk test. Rev Port Pneumol. 2009;15(1):11-25. doi: http://dx.doi.org/10.1016/s2173-5115(09)70085-5

41. Brown CD, Benditt JO, Sciurba FC, et al. Exercise testing in severe emphysema: Association with quality of life and lung function. COPD. 2008;5(2):117-124. doi: http://dx.doi.org/10.1080/15412550801941265

42. Camargo LA, Pereira CA. Dyspnea in COPD: beyond the modified Medical Research Council scale. J Bras De Pneumol. 2010;36(5):571-758.
43. Carter R, Holiday DB, Nwasuruba C, et al. 6-minute walk work for assessment of functional capacity in patients with COPD. Chest. 2003; 123(5) :1408-1415.

44. Chen S, Wu YT, Lin JJ, Lee CN, Huang CY, Chiang LL. The correlations of the six-minute walk test and respiratory functions in chronic obstructive pulmonary disease patients with chronic hypercapnia. J Exp Clin Med. 2012;4(1):47-51. doi: http://dx.doi.org/10.1016/j.jecm.2011.11.008

45. Chetty KG, Brown SE, Milne N, Light RW. Right ventricular dysfunction and its relationship to maximal oxygen consumption of patients with chronic obstructive pulmonary disease. J Cardiopulm Rehabil. 1989;9(5):183-187. doi: http://dx.doi.org/10.1097/00008483-198905000-00001

46. Chuang ML, Lin IF, Wasserman K. The body weight-walking distance product as related to lung function, anaerobic threshold and peak VO2 in COPD patients. Respir Med. 2001;95(7):618626. doi: http://dx.doi.org/10.1053/rmed.2001.1115

47. Cote CG, Pinto-Plata V, Kasprzyk K, Dordelly LJ, Celli BR. The 6-min walk distance, peak oxygen uptake, and mortality in COPD. Chest. 2007;132(6):1778-1785. doi: http://dx.doi.org/10.1378/chest.07-2050

48. Dekhuyzen PNR, Kaptein AA, Dekker FW. Twelve-minute walking test in a group of Dutch patients with chronic obstructive pulmonary diseases: Relationship with functional capacity. Eur J Respir Dis. 1986;69(Suppl. 146):259-264.

49. Dillard TA, Piantadosi S, Rajagopal KR. Determinants of maximum exercise capacity in patients with chronic airflow obstruction. Chest. 1989;96(2):267-271.

doi: http://dx.doi.org/10.1378/chest.96.2.267

50. Dowson LJ, Newall C, Guest PJ, Hill SL, Stockley RA. Exercise capacity predicts health status in alpha1-antitrypsin deficiency. Am J Respir Crit Care Med. 2001;163(4):936-941. doi: http://dx.doi.org/10.1164/ajrccm.163.4.2007048

51. Emtner MI, Arnardottir HR, Hallin R, Lindberg E, Janson C. Walking distance is a predictor of exacerbations in patients with chronic obstructive pulmonary disease. Respir Med. 2007;101(5):1037-1040. doi: http://dx.doi.org/10.1016/j.rmed.2006.09.020

52. Fujimoto $H$, Asai $K$, Watanabe $T$, Kanazawa $H$, Hirata $K$. Association of six-minute walk distance (6MWD) with resting pulmonary function in patients with chronic obstructive pulmonary disease (COPD). Osaka City Med J. 2011;57(1):21-29.

53. Fujita E, Nagasaka Y, Kozuka T, Ebara H, Fukuoka M. Correlation among the indices of high-resolution computed tomography, pulmonary function tests, pulmonary perfusion scans and exercise tolerance in cases of chronic pulmonary emphysema. Respiration. 2002;69(1):30-37. doi: http://dx.doi.org/10.1159/000049367

54. Gosselink R, Troosters T, Decramer M. Peripheral muscle weakness contributes to exercise limitation in COPD. Am J Respir Crit Care Med. 1996;153(3):976-980. doi: http://dx.doi.org/10.1164/ajrccm.153.3.8630582

55. Hill K, Jenkins SC, Cecins N, Philippe DL, Hillman DR, Eastwood PR. Estimating maximum work rate during incremental cycle ergometry testing from six-minute walk distance in patients with chronic obstructive pulmonary disease. Arch Phys Med Rehabil. 2008;89(9):1782-1787.

doi: http://dx.doi.org/10.1016/j.apmr.2008.01.020 
56. Hodgev VA, Aliman OI, Marinov BI, Kostianev SS, Mandulova PV. Cardiovascular and dyspnea response to six-minute and shuttle walk tests in COPD patients. Folia Med. 2003;45(3):26-33.

57. Kozu R, Jenkins S, Senjyu H, Mukae H, Sakamoto H, Kohno S. Peak power estimated from 6-minute walk distance in Asian patients with idiopathic pulmonary fibrosis and chronic obstructive pulmonary disease. Respirology. 2010;15(4):706-713. doi: http://dx.doi.org/10.1111/j.1440-1843.2010.01744.x

58. Light RW, Merrill EJ, Despars JA. Prevalence of depression and anxiety in patients with COPD. Relationship to functional capacity. Chest. 1985;87 (1):35-38. doi: http://dx.doi.org/10.1378/chest.87.1.35

59. McGavin CR, Gupta SP, McHardy GJR. Twelve minute walking test for assessing disability in chronic bronchitis. BMJ. 1976;1 (6013):822-823. doi: http://dx.doi.org/10.1136/bmj.1.6013.822

60. Morgan AD, Peck DF, Buchanan DR, McHardy GJR. Effect of attitudes and beliefs on exercise tolerance in chronic bronchitis. BMJ. 1983;286 (6360):171-173. doi: http://dx.doi.org/10.1136/bmj.286.6360.171

61. Mungall IPF, Hainsworth R. Assessment of respiratory function in patients with chronic obstructive airways disease. Thorax. 1979;34 (2):254-258. doi: http://dx.doi.org/10.1136/thx.34.2.254

62. Nakamura Y, Tanaka K, Shigematsu R, Homma T, Sekizawa $\mathrm{K}$. Determinants of cardiorespiratory fitness in patients with chronic obstructive pulmonary disease, focusing on activities parallel to daily living. Respirology. 2004;9(3):326-330. doi: http://dx.doi.org/10.1111/j.1440-1843.2004.00605.x

63. Oga T, Nishimura K, Tsukino M, Hajiro T, Ikeda A, Mishima M. Relationship between different indices of exercise capacity and clinical measures in patients with chronic obstructive pulmonary disease. Heart Lung. 2002;31(5):374-381. doi: http://dx.doi.org/10.1067/mhl.2002.127941

64. O'Reilly JF, Shaylor JM, Fromings KM, Harrison BD. The use of the 12 minute walking test in assessing the effect of oral steroid therapy in patients with chronic airways obstruction. British $J$ Dis Chest.1982; 76(4):374-382. doi: http://dx.doi.org/10.1016/0007-0971(82)90073-0

65. Peruzza S, Sergi G, Vianello A et al. Chronic obstructive pulmonary disease (COPD) in elderly subjects: Impact on functional status and quality of life. Respir Med. 2003;97(6):612617. doi: http://dx.doi.org/10.1053/rmed.2003.1488

66. Rejeski WJ, Foley KO, Woodard CM, Zaccaro DJ, Berry MJ. Evaluating and understanding performance testing in COPD patients. J Cardiopul Rehabil. 2000;20(2):79-88. doi: http://dx.doi.org/10.1097/00008483-200003000-00001

67. Revill SM, Morgan MDL, Singh SJ, Williams J, Hardman AE. The endurance shuttle walk: A new field test for the assessment of endurance capacity in chronic obstructive pulmonary disease. Thorax. 1999;54(3):213-22.

doi: http://dx.doi.org/10.1136/thx.54.3.213

68. Schols AMWJ, Mostert R, Soeters PB, Greve LH, Wouters EFM. Nutritional state and exercise performance in patients with chronic obstructive lung disease. Thorax. 1989;44(11):937-941. doi: http://dx.doi.org/10.1136/thx.44.11.937
69. Singh SJ, Morgan MDL, Scott S, Walters D, Hardman AE. Development of a shuttle walking test of disability in patients with chronic airways obstruction. Thorax. 1992;47(12):1019-1024. doi: http://dx.doi.org/10.1136/thx.47.12.1019

70. Wakayama K, Kurihara N, Fujimoto S, Hata M, Takeda T. Relationship between exercise capacity and the severity of emphysema as determined by high resolution CT. Eur Respir J. 1993;6(9):1362-1367.

71. Yoshikawa M, Yoneda T, Kobayashi A, et al. Body composition analysis by dual energy $\mathrm{x}$-ray absorptiometry and exercise performance in underweight patients with COPD. Chest. 1999;115(2):371-375. doi: http://dx.doi.org/10.1378/chest.115.2.371

72. Wijkstra PJ, TenVergert EM, van der Mark TW, et al. Relation of lung function, maximal inspiratory pressure, dyspnoea, and quality of life with exercise capacity in patients with chronic obstructive pulmonary disease. Thorax. 1994; 49(5): 468-472. doi: http://dx.doi.org/10.1136/thx.49.5.468

73. Pitta F, Takai MY, Ovierira NH, et al. Relationship between pulmonary function and physical activity in daily life in patients with COPD. Respir Med. 2008; 102(8): 1203-1207. doi: http://dx.doi.org/10.1016/j.rmed.2008.03.0004

74. Chen LF, Wang CH, Chou PC, et al. Association between emphysema score, six-minute walk and cardiopulmonary exercise tests in COPD. Open Respir Med J. 2012;6(1):104-110. doi: http://dx.doi.org/10.2174/1874306401206010104

75. Waatevik M, Johannessen A, Hardie JA, et al. Different COPD disease characteristics are related to different outcomes in the 6-minute walk test. COPD. 2012;9(3):227-234. doi: http://dx.doi.org/10.3109/15412555.2011.650240

76. Vagaggini B, Taccola M, Severino S, et al. Shuttle walking test and 6-minute walking test induce a similar cardiorespiratory performance in patients recovering from an acute exacerbation of chronic obstructive pulmonary disease. Respiration. 2003;70(6):579-584. doi: http://dx.doi.org/10.1159/000075202

77. Rosa FW, Camelier A, Mayer A, Jardim JR. Evaluating physical capacity in patients with chronic obstructive pulmonary disease: Comparing the shuttle walk test with the encouraged 6-minute walk test. [Portuguese, English]. J Bras Pneumol. 2006;32(2):106113. doi: http://dx.doi.org/10.1590/S1806-37132006000200005

78. Chonan T, Hida W, Kikuchi Y, Shindoh C, Takishima T. Role of $\mathrm{CO}_{2}$ responsiveness and breathing efficiency in determining exercise capacity of patients with chronic airway obstruction. Am Rev Respir Dis. 1988;138(6):1488-1493. doi: http://dx.doi.org/10.1164/ajrccm/138.6.1488

79. Pitta F, Takaki MY, Oliveira NHd, et al. Relationship between pulmonary function and physical activity in daily life in patients with COPD. RespirMed. 2008;102 (8):1203-1207. doi: http://dx.doi.org/10.1016/j.rmed.2008.03.004

80. Baarends EM, Schols AMWJ, Mostert R, Wouters EFM. Peak exercise response in relation to tissue depletion in patients with chronic obstructive pulmonary disease. Eur Respir J. 1997;10(12):2807-13.

doi: http://dx.doi.org/10.1183/09031936.97.10122807 
81. Martinez FJ, Foster G, Curtis JL, et al. Predictors of mortality in patients with emphysema and severe airflow obstruction. Am J Respir Crit Care Med. 2006;173(12):1326-1334. doi: http://dx.doi.org/10.1164/rccm.200510-16770C

82. Casanova C, Cote C, Marin JM, et al. Distance and oxygen desaturation during the 6-min walk test as predictors of longterm mortality in patients with COPD. Chest. 2008;134(4):746752. doi: http://dx.doi.org/10.1378/chest.08-0520

83. Martin C, Chapron J, Hubert D, et al. Prognostic value of six minute walk test in cystic fibrosis adults. Respir Med. 2013;107(12):1881-1887.

doi: http://dx.doi.org/10.1016/j.rmed.2013.10.001

84. Celli BR, Cote CG, Marin JM, et al. The body-mass index, airflow obstruction, dyspnea, and exercise capacity index in chronic obstructive pulmonary disease. NEngl J Med. 2004;350(10):10051012. doi: http://dx.doi.org/10.1056/NEJMoa021322

85. Holland AE, Spruit MA, Troosters T, et al. An official European Respiratory Society/American Thoracic Society technical standard: field walking tests in chronic respiratory disease. Eur Respir J. 2014;44(6):1428-1446. doi: http://dx.doi.org/10.1183/09031936.00150314

86. Turner SE, Eastwood PR, Cecins NM, Hillman DR, Jenkins SC. Physiologic responses to incremental and self-paced exercise in COPD: A comparison of three tests. Chest. 2004;126(3):766-773. doi: http://dx.doi.org/10.1378/chest.126.3.766

87. Solway S, Brooks D, Lacasse Y, Thomas S. A qualitative systematic overview of the measurement properties of functional walk tests used in the cardiorespiratory domain. Chest. 2001;119(1):25 